NURSE DECISION-MAKING IN ACUTE CARE

by

Christine Williams Nibbelink

$\overline{\text { Copyright @ Christine Williams Nibbelink } 2017}$

\author{
A Dissertation Submitted to the Faculty of the \\ COLLEGE OF NURSING \\ In Partial Fulfillment of the Requirements \\ For the Degree of \\ DOCTOR OF PHILOSOPHY \\ In the Graduate College \\ THE UNIVERSITY OF ARIZONA
}

2017 


\section{THE UNIVERSITY OF ARIZONA}

GRADUATE COLLEGE

As members of the Dissertation Committee, we certify that we have read the dissertation prepared by Christine Williams Nibbelink entitled "Nurse Decision-Making in Acute Care" and recommend that it be accepted as fulfilling the dissertation requirement for the Degree of Doctor of Philosophy

Date: April 14, 2017

Jane M. Carrington, $\mathrm{PhD}, \mathrm{RN}$

Date: $\underline{\text { April 14, } 2017}$

Barbara B. Brewer, PhD, RN, MALS, MBA, FAAN

Date: April 14, 2017

Kimberly D. Shea, PhD, RN, CHPN

Final approval and acceptance of this dissertation is contingent upon the candidate's submission of the final copies of the dissertation to the Graduate College.

I hereby certify that I have read this dissertation prepared under my direction and recommend that it be accepted as fulfilling the dissertation requirement.

Dissertation Director: Jane M. Carrington, PhD, RN

Date: April 14, 2017 


\section{STATEMENT BY AUTHOR}

This dissertation has been submitted in partial fulfillment of requirements for an advanced degree at The University of Arizona and is deposited in the University Library to be made available to borrowers under rules of the Library.

Brief quotations from this dissertation are allowable without special permission, provided that accurate acknowledgment of source is made. Requests for permission for extended quotation from or reproduction of this manuscript in whole or in part may be granted by the copyright holder

SIGNED: _ Christine Williams Nibbelink 


\section{ACKNOWLEDGMENTS}

The pursuit of a Doctor of Philosophy in Nursing comes with tremendous support. This achievement is by no means a result of my efforts alone. I am grateful to so many.

My Dissertation Committee:

Jane M. Carrington, $\mathrm{PhD}, \mathrm{RN}$, committee chair. You have gone above and beyond in your support of my research and growth as a nursing scientist. Your fresh view on all things nursing informatics is truly inspiring.

Barbara B. Brewer, PhD, RN, MALS, MBA, FAAN, committee member. You have provided me with a consistent source of support for my nursing science questions and have held me to a high standard I hope to continue throughout my career.

Kimberly D. Shea, PhD, RN, CHPN, committee member. Your knowledge of informatics was essential to my progress as a nursing scientist. I also greatly appreciate your consistently open attitude to all questions over the years.

Thank you also to the scholarship finding from the June and Walter Reis Endowed Scholarship and the Lange Award for the support of this research.

To my husband, Kevin Nibbelink, thank you is not enough for the constant support and encouragement you have provided as I have worked toward my goal of completing this degree.

To my children Luke, Megan, Ethan, you also have been wonderful throughout this process. I am so proud of each of you. 


\section{TABLE OF CONTENTS}

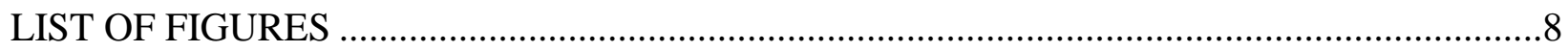

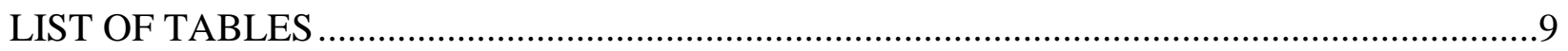

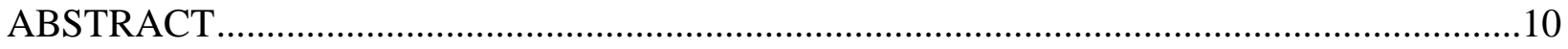

CHAPTER I: REVIEW OF THE LITERATURE .................................................... 11

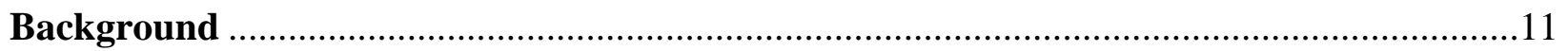

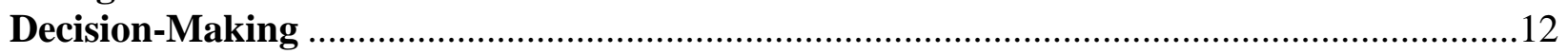

Electronic Health Record and Clinical Decision Support Systems ..................................19

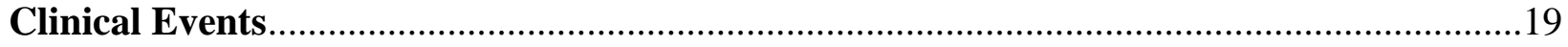

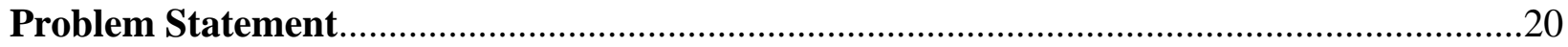

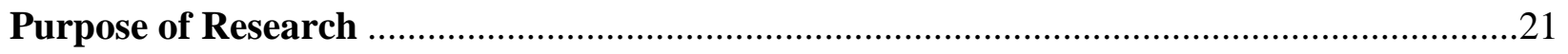

Research Definitions .........................................................................................21

Research Questions ..........................................................................................22

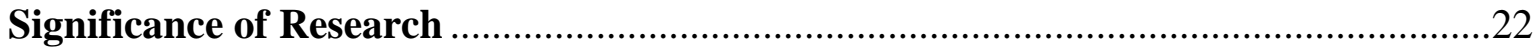

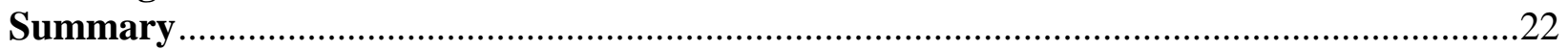

CHAPTER II: CONCEPTUAL FRAMEWORK .......................................................24

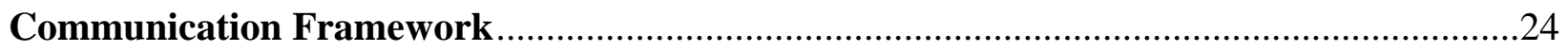

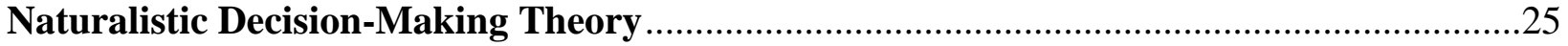

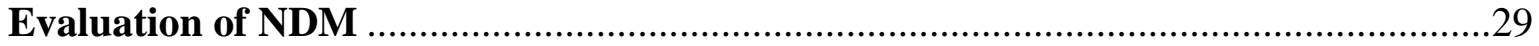

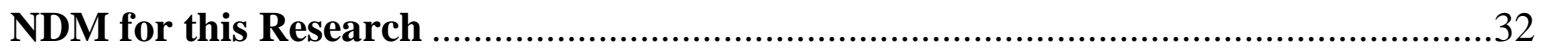

NDM and Communication Framework for this Research ........................................34

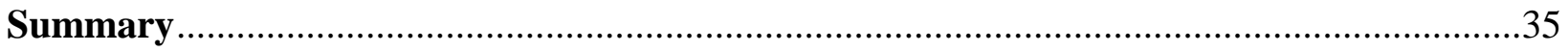

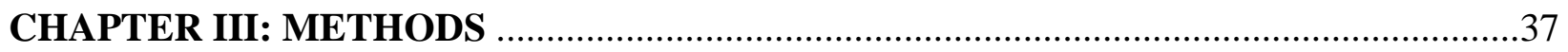

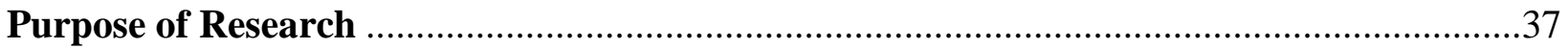

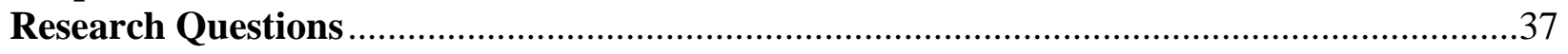

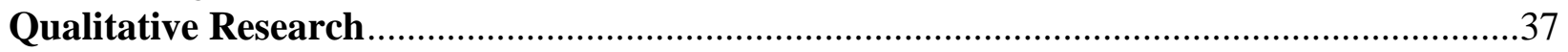

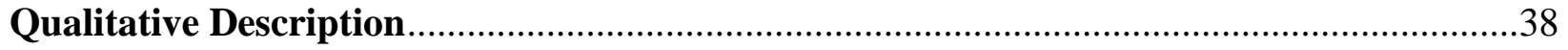

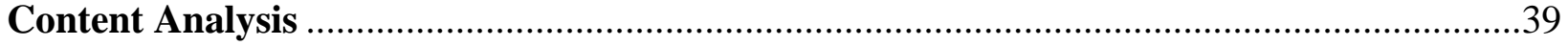

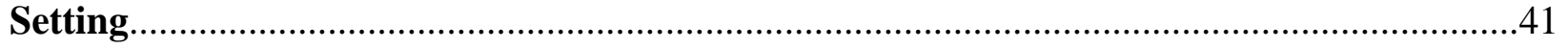

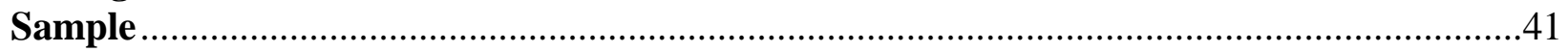

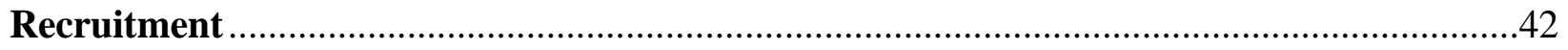

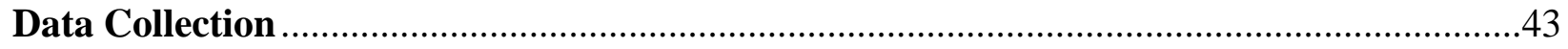

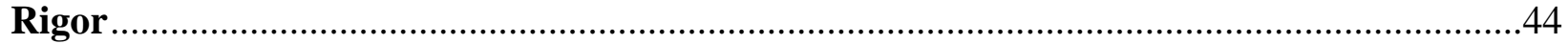

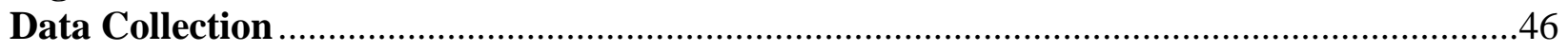

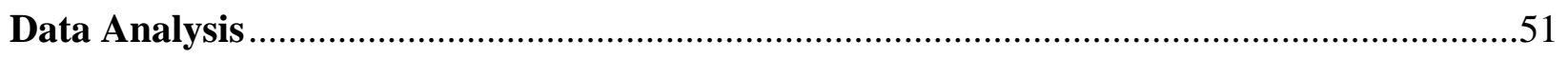

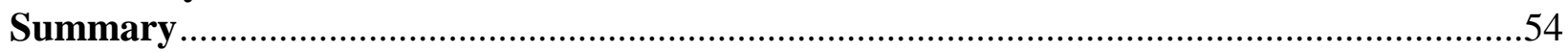


TABLE OF CONTENTS - Continued

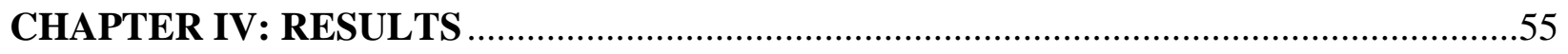

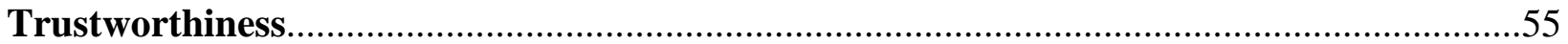

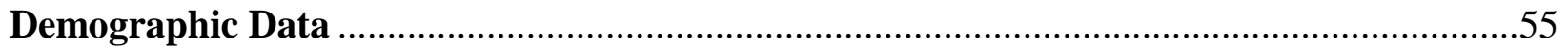

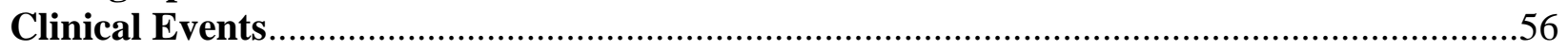

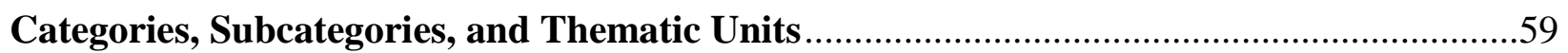

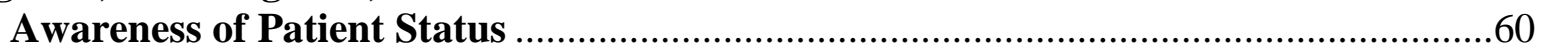

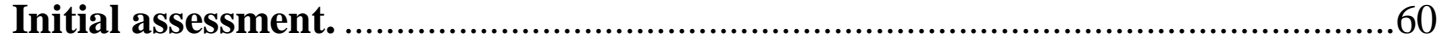

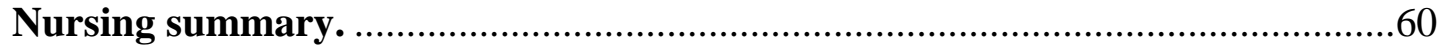

Patient factors that support decision-making ...................................................60

Awareness of change in patient status. .........................................................61

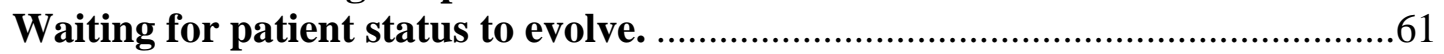

Identification of increased patient need.............................................................61

Nursing report to next shift or physician .........................................................61

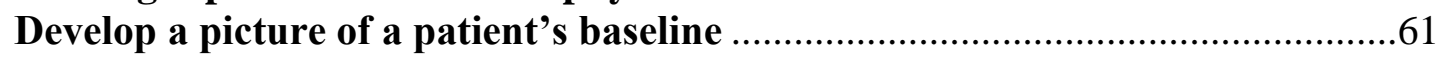

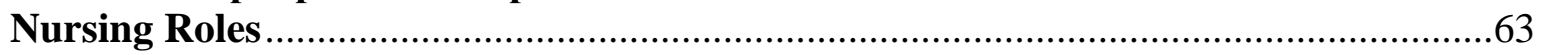

Factors that conflict with RN ideal goals.......................................................63

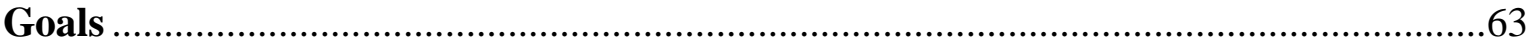

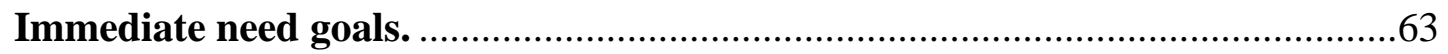

Goals for the present shift/prior to discharge..................................................64

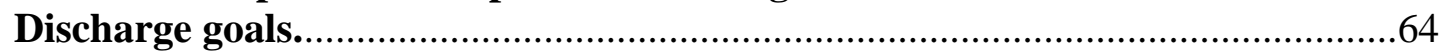

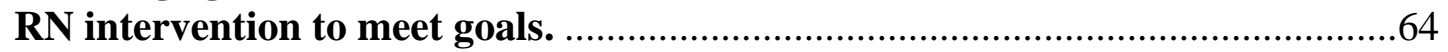

Education/Certification/Hospital Training to Support Decision-Making ....................65

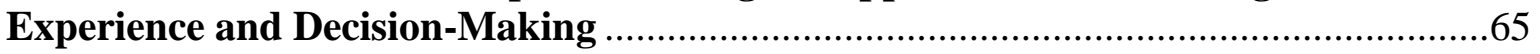

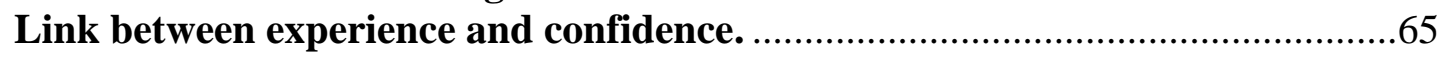

Previous experience with similarities or differences in patients that influence decision-making. .........................................................................................65

Experience and education in decision-making...................................................65

Experience influence on decision-making/nursing practice. .................................66

Reflecting to when inexperienced/development of confidence through

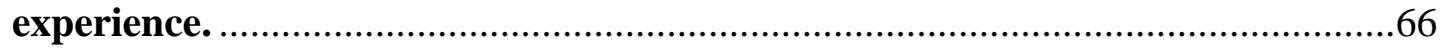

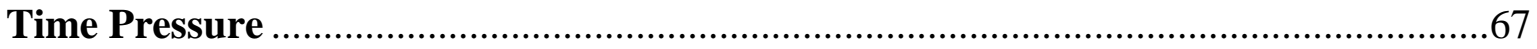

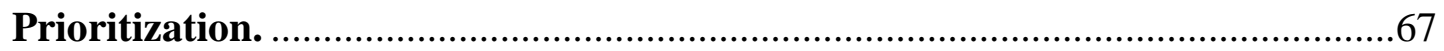

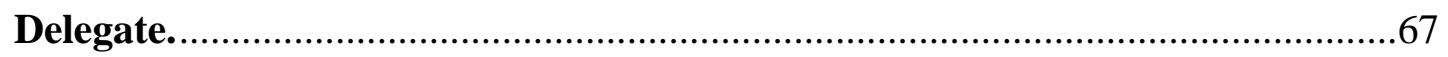

Patient care prioritization based on stability …………....................................67

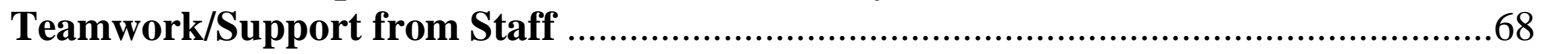

Teamwork/support from staff for decision-making. ...........................................68

Support from staff to complete patient care. …………………………………....68

Additional support options considered to meet goals...........................................68

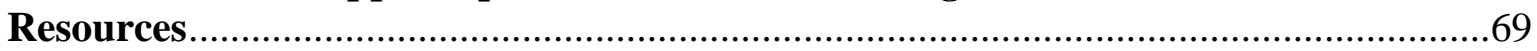

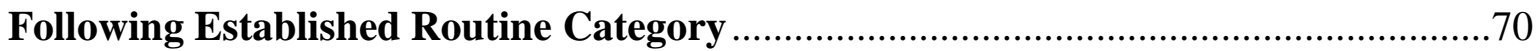

Describe viewing patient as individual routinely................................................70 
TABLE OF CONTENTS - Continued

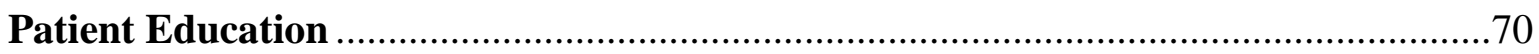

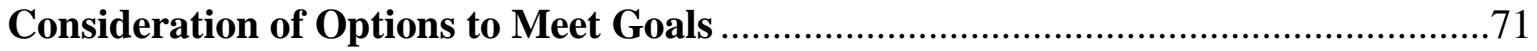

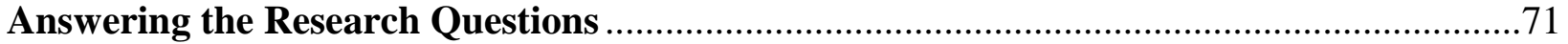

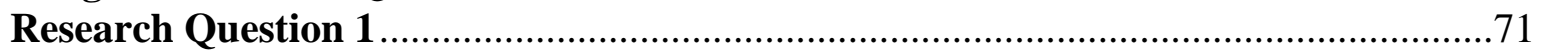

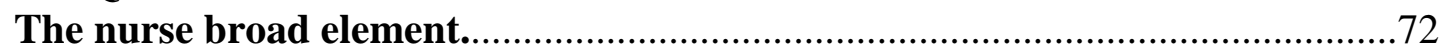

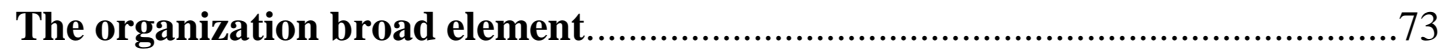

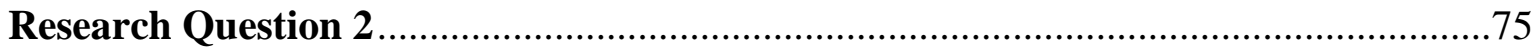

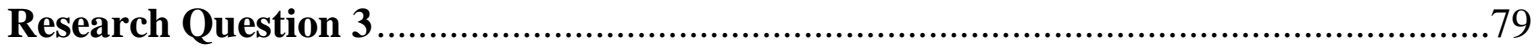

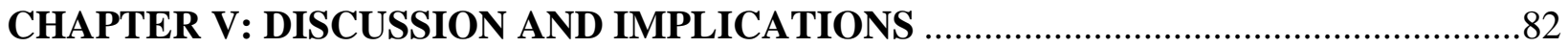

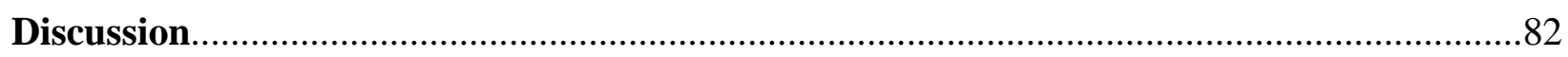

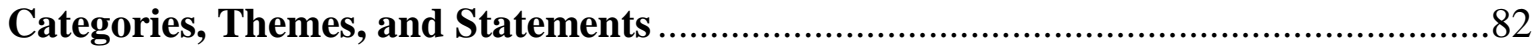

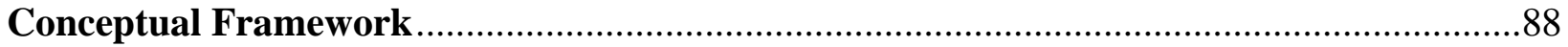

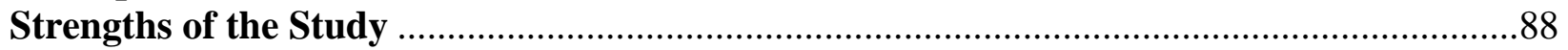

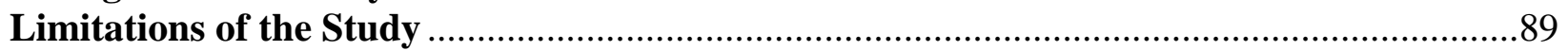

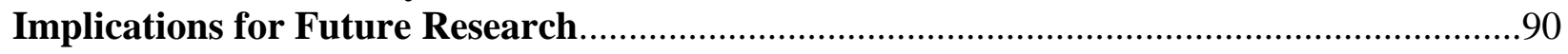

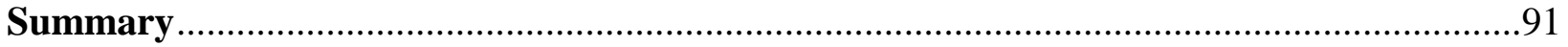

APPENDIX A: DATA COLLECTION INTERVIEW GUIDE.............................................93

APPENDIX B: UA IRB APPROVAL LETTER CEDED OVERVIEW ..............................97

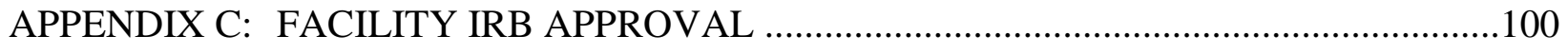

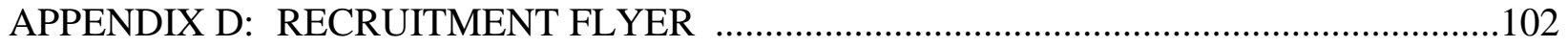

APPENDIX E: APPROVED CONSENT FOR RESEARCH ….........................................104

APPENDIX F: THANK YOU LETTER TO PARTICIPANTS ..........................................108

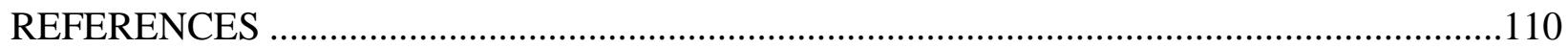




\section{LIST OF FIGURES}

FIGURE 1. Naturalistic Decision Making Based on Literature ...........................................28

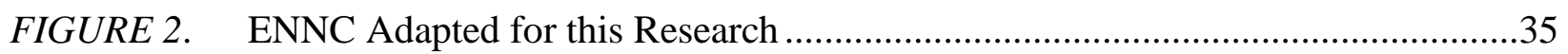




\section{LIST OF TABLES}

TABLE 1. Responding and Receiving Nurses Interview Questions Aligned with Research Questions..............................................................................................

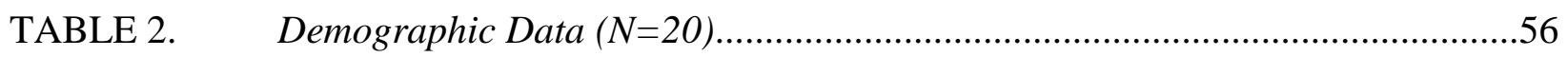

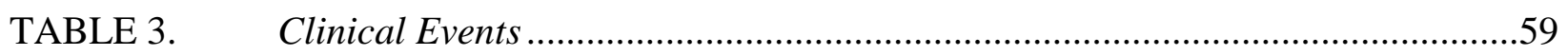

TABLE 4. Awareness of Patient Status Category …………………………....................61

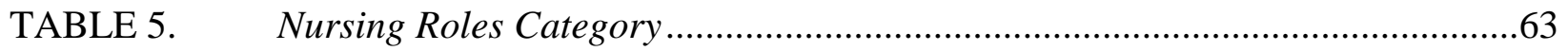

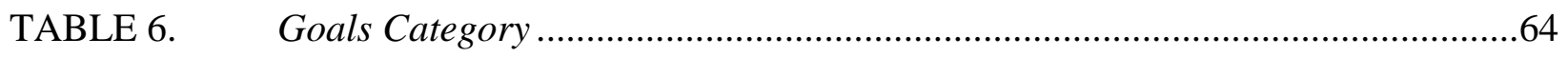

TABLE 7. Education/Certification/Hospital Training to Support Decision-Making

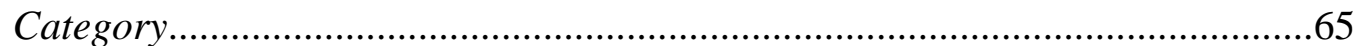

TABLE 8. $\quad$ Experience and Decision-Making Category .......................................................66

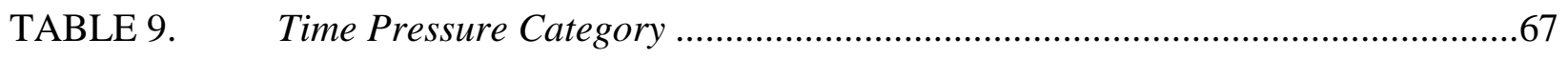

TABLE 10. Teamwork/Support from Staff Category ……....................................................69

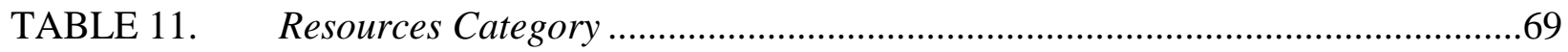

TABLE 12. Following Established Routine Category ........................................................

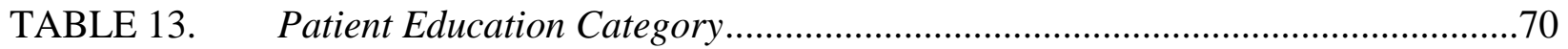

TABLE 14. Consideration of Options to Meet Goals Category ………………….................

TABLE 15. Comparison of Responding and Receiving Nurses and Decision-Making .........79

TABLE 16. $\quad$ Novice and Experienced Nurse Responses .........................................................81 


\begin{abstract}
The Institute of Medicine identified that 98,000 deaths occur in hospitals each year due to poor decision-making. The need for better understanding of decision-making in patient care is evident. The purpose of this study is to explore nurses' perceptions of clinical decision-making for a patient who experienced a clinical event. The overarching framework for this research is the Effective Nurse to Nurse Communication framework which uses clinical events, or sudden changes in patient condition, to explore nurse communication between nurses who respond to a clinical event and nurses who receive report from the responding nurse about the clinical event. Naturalistic Decision Making is the framework used to explore the decision-making factors used by experienced decision makers in real world conditions. These frameworks provided the basis for data collection, interview question development, and facilitated data analysis. Twenty nurses in an urban acute care hospital were interviewed at their workplace about a patient who had experienced a sudden change in condition in the previous 24 hours. These interviews were transcribed and analyzed using content analysis. Categories that emerged were: Awareness of Patient Status, Nursing Roles (outside of specific patient care), Goals, Education / Certification / Hospital Training to support decision-making, Experience and Decision-Making, Time Pressure, Teamwork / Support from Staff, Resources, Following Established Routine. Patient Education, and Consideration of Options to Meet Goals. Further analysis indicates that elements of decisionmaking differ between responding and receiving nurses and based on experience level of the nurse. Strengths, limitations, and suggestions for future research are presented.
\end{abstract}




\section{CHAPTER I: REVIEW OF THE LITERATURE}

Research by the Institute of Medicine suggests that 98,000 deaths occur in hospitals each year as a result of poor decision-making (Kohn, Corrigan, \& Donaldson, 1999). Decision making for acute care nurses is highly demanding. For instance, critical care nurses make 238 decisions every two hours resulting in one decision every 30 seconds or 1,428 per 12-hour shift (Bucknall 2000). The importance of effective decision-making in acute care cannot be overemphasized. Incorporating evidence based patient care is essential for ideal patient outcomes (Yancey et al., 2013). In addition, responses to patient crises must be effective and occur in a time efficient manner to enhance patient survival (Sandroni et al., 2004). The decision-making process for acute care nurses is complex. This process includes and guides identification of patterns and similarities among experiences (Benner \& Tanner, 1987). Research designed to increase our understanding of nurse pattern identification and similarities among experiences will inform strategies to increase effective nurse decision-making and result in improved patient outcomes. The purpose of this research is to explore factors that influence nurse decision making in the acute care setting. Chapter 1 includes a discussion of current decision making literature and rationale for this research based on a gap in the body of nursing knowledge related to decision making in actual settings as well as a description of clinical events in acute care hospital settings.

\section{Background}

Clinical decision-making is synonymous with clinical judgment and includes evaluation and interpretation of patient assessment findings potentially resulting in nursing intervention (Tanner, 2006). Traditionally, Classical Decision Making (CDM) has guided research in nurse decision-making (Cioffi, 2012). This model requires the decision maker to choose from a list of 
options based on predictions made by the decision maker related to which option will meet goals (Lipshitz, Klein, Orasanu, \& Salas, 2001). The CDM process includes a comprehensive review of options with a distinct selection of one option (Lipshitz et al., 2001). The limitation of CDM is that it takes too much time in real world (non-laboratory) settings that include time limits and other factors (Klein \& Calderwood, 1991). Decision-making in acute care nursing occurs in complex circumstances and involves many factors identified in research. These factors include a nursing consideration of not just the needs of the patient but numerous other factors when making decisions (Klein, Calderwood, \& Clinton-Cirocco, 2010). Factors identified as important to nurse decision making in this literature review include experience, culture, education, knowledge, situation awareness, autonomy, time pressure, physician issues, and models used in decision making.

\section{Decision-Making}

-Experience provides several influencing factors for nurses in acute care. For instance, experience can lead to increased self-confidence which influences nursing skills, nursing diagnosis, nursing interventions and identification of patient needs (Fry \& MacGregor, 2014; Radwin, 1998). Self-confidence also increases nurses' individualized interventions for patient care in order to enhance patient specific care (Radwin, 1998). However, experience alone does not lead to improved clinical decision making (Agbedia, Ofi, \& Ibeagha, 2008; Sedgwick, Grigg, \& Dersch, 2014; Stubbings, Chaboyer, \& McMurray, 2012; Thompson et al., 2009). For instance, nurses' interventions, situation awareness, and effective clinical judgment are not enhanced through increased experience in nursing (Agbedia et al., 2008; Sedgwick et al., 2014; Stubbings et al., 2012; Thompson et al., 2009). In other research, expertise did facilitate factors 
associated with decision-making. Expertise did include instinct, pattern identification in their practice, and improved critical thinking (Bakalis, 2013; Martin, 2002; Rycroft-Malone, 2009). Nurses describe seeking the support of colleagues for decision-making and do not necessarily focus on the information the colleague provides. This support includes an informal evaluation of the colleague's experience, role, and an evaluation of the colleague's response social and supportive reasons (Rycroft-Malone et al., 2009; Seright, 2011; Marshall et al., 2013; Offredy, Kendall, \& Goodman, 2008). Nurses perceive the personal evaluation of individual colleagues as more important than the information received (Marshall et al., 2013).

The perceived value of the experienced nurse as a decision support system has a strong influence on decision-making. Nurses believe that experienced nurses can provide more applicable advice than protocols (Cappelletti et al., 2014; Husted, 2001; Marshall, West, \& Aitken, 2013; Rycroft-Malone et al., 2009; Samuriwo \& Dowding, 2014; Seright, 2011). Nurses do find evidence to be helpful to nursing practice. Evidence based protocols have been described as facilitating decision making for novices, providing a resource for decision making in uncertain circumstances, and enhancing patient safety (Bakalis, 2013; Dougherty, Sque, \& Crouch, 2012; Fry \& MacGregor, 2014; Rycroft-Malone et al., 2009). However, referencing protocols can be difficult in time-limited situations (Rycroft-Malone et al., 2009). In addition, nurses may find that a protocol does not apply to their patient care situation leading them to use their own ideas for individualized patient care practice (Dougherty, Sque, \& Crouch, 2012; Downing, Yoder, \& Kirksey, 2011; Samuriwo \& Dowding, 2014;). While nurses believe that evidence is important for nursing practice, they identify several barriers that interfere with its implementation in acute care nursing decision-making. 
Numerous studies suggest that the culture of an organization can influence decision making in nursing practice (Braaten, 2015; Cappelletti et al., 2014; Hendry \& Walker, 2004; Jezewski, 1994; Kihlgren, Forslund, \& Fagerberg, 2006; Krairiksh \& Anthony, 2001; Mantzoukas \& Jasper, 2004; Offredy et al., 2008; Stubbings et al., 2012). Nurses identify clinical nursing environments that include transparent patient goals as supportive (Kihlgren et al., 2006). However, when disagreement among healthcare professionals exists in the patient care setting, nurses describe difficulty in decision-making (Jezewski, 1994). Therefore, fostering a positive nursing practice culture is important to improved decision-making. Unwritten practices may also influence nurse decision-making. Examples include calling rapid response teams, patient care planning, and exchange of information in the patient care environment (Braaten, 2015; Cappelletti et al., 2014; Gray \& Meyer, 2014). Unwritten practices that only the nurses' familiar with the culture of the unit understand make decision-making for new or floating nurses more challenging. Various levels of decision-making can affect unit culture. For instance, situation awareness, "an understanding of the state of the environment" (Endsley, 1995, p. 65), varies based on leadership, personality differences, and nurse manager relationships (Mantzoukas \& Jasper, 2004; Stubbings et al., 2012). Not all nurses influence decision making equally. One study identified that the "right nurse" must present changes in order to have the changes accepted (Mantzoukas \& Jasper, 2004, p. 930). Unpredictable and informal pressures within the nursing practice environment heavily influence nursing decision-making creating concern for the strength and communication of unwritten practices in nursing practice.

Nursing education programs have inconsistent effects on decision making in nursing practice. One research study found a lack of significant difference in critical thinking between 
associate and bachelor degree prepared nurses (Martin, 2002). Education designed to improve critical thinking in the clinical setting also did not lead to improved decision making (DohertyKing \& Bowers, 2013; Thompson \& Stapley, 2011). In other research, critical thinking did improve among masters and baccalaureate prepared nurses as compared to associate degree prepared nurses (Pardue, 1987). Specific responses in acute care were different based on education. Nurses from four-year programs activated emergency medical teams more frequently than nurses from two-year programs (Pantazopoulos et al., 2012). Because research identifies a wide variation in the association among education, decision-making, and critical thinking, the connection between these concepts must be further explored.

Knowledge of the patient, patient assessment, and situation awareness were elements associated with decision-making research. Patient specific information is inconsistently perceived as important to decision making. For instance, "knowing the patient" included an understanding of patient patterns and is described as useful but not the focus of decision making (Cappelletti et al., 2014, p. 454; Sedgwick et al., 2014). Other research finds that nurses may use patient specific information to categorize patients when making decisions (Hodge, Hugman, Varndell, \& Howes, 2013; Tower, Chaboyer, Green, Dyer, \& Wallis, 2012). Similarly, nurses find that patient circumstances may trigger a memory of a previous patient care experience which may help to diminish uncertainty in decision making (French, 2006). One study found that $50 \%$ of influence in nursing decision-making is based on two cues: dizziness and respiratory rate (Thompson et al., 2009). Another study indicated that heart rate, thoracic pain, airway obstruction, and respiratory rate were most important to nurses when making decisions 
(Pantazopoulos et al., 2012). Patterns and memories of previous patient care situations appear to influence decision-making.

Situation awareness involves understanding the current state in order to make decisions (Endsley, 1997). This understanding of a patient's current state is an essential factor in the decision-making process for acute care nurses in order to provide effective patient care. Nurses use patient diagnoses, their understanding of the significance of a patient's current situation, and prediction of possible outcomes when deciding on a plan of care (Tower et al., 2012). Situation awareness, for this process, is influenced by various factors. Self-confidence and assertiveness influence individual nursing situation awareness but experience does not (Stubbings et al., 2012). Nurses identify confidence as a resource for decision making (Fry \& MacGregor, 2014). However, nurses with lower self-confidence are more accurate in responses than those with high self-confidence (Fonseca \& Brennan, 2012). The inverse relationship between high selfconfidence and accuracy leads to concern that potentially those who are confident in their responses may be making poor decisions. Future research could help identify the importance of the relationship between confidence and decision-making.

The influence of autonomy is important for nurse decision-making. Nurses identify feeling autonomous when patient care is conducted using their expertise and, counter intuitively, interdependently (Stewart, Stansfield, \& Tapp, 2004). Actions described as associated with autonomy include patient advocacy, discussions with physicians, education of patients related to medication regimen, and preservation of skin integrity (Mrayyan, 2004). Other factors linked with autonomy include education, clinical experience, specialty focus, shift worked, and hospital size (Holl, 1996). Nurses experience a feeling of diminished autonomy when they do not feel 
acknowledged, are recently graduated, informing patients about surgical risks, ordering diagnostic tests, or determining the date of discharge (Doherty-King \& Bowers, 2013; Mrayyan, 2004; Stewart et al., 2004). It is interesting to note that some of the actions associated with lower autonomy are outside the nurse's scope of practice. Support and encouragement for nurses to practice within their scope of practice could potentially increase autonomy for some nurses.

Time limited patient care situations led to challenges in decision-making. Time pressure significantly decrease decision-making proficiency (Thompson et al., 2008). Experienced critical care nurses made accurate decisions when not time limited as compared to inexperienced nurses but this difference was not present when time limits were present (Thompson et al., 2008). This limitation in the value of experience during time pressured situations may indicate an important opportunity for support in nursing practice. The presence of time limitations influenced nurse decision-making. For instance, the amount of time available influenced the selection of patient interventions, prioritization of patient care, and the extensiveness of patient assessment when deciding to call a rapid response team (Braaten, 2015; Hedberg \& Larsson, 2003; Hendry \& Walker, 2004). Nurses also described that a patient whose status was rapidly deteriorating decreased the perceived need for extensive assessment while a more gradual decline led nurses to spend more time in assessment (Braaten, 2015). The increased time spent assessing a patient experiencing a gradual deterioration in status included consulting with more experienced colleagues and collecting additional information to justify decision-making (Braaten, 2015). Time clearly influences nurses' decision-making when providing patient care.

Nurse decision-making is also influence by nurse-physician collaborative dynamics. Nurses have reported that their involvement in shared decision-making with physicians increases 
when a trusting collaborative relationship (nurse-physician) is fostered within the environment. (Krairiksh \& Anthony, 2001; Merrick, Fry, \& Duffield, 2014). Nurses describe strategies to establish the trusting relationship by flattering physicians (Gallagher et al., 2015; Merrick et al., 2014). The influence of physicians on nursing decision-making appears to include a social element that may be distracting to the decision-making process.

Decision models are useful to describe how decisions are made in nursing practice. For example, analytic decision makers develop and test a hypothesis compared to intuitive decision makers, who do not use a conscious rationale for decision-making (Cappelletti et al., 2014; Parker, 2014). These models may be used together in some nursing practice situations (Cappelletti et al., 2014; Parker, 2014). The results using these models are inconclusive. Only $35 \%$ of decisions made intuitively coincided with decisions made analytically (Panniers \& Walker, 1994). Possibly, these observed differences in both decision-making models implemented by nurses and decision-making outcomes may lead to differences in patient outcomes.

Review of literature suggests that there are many important unanswered questions related to decision making in acute care nursing. A better understanding of the influence of experience, education and knowledge, organizational culture, situation awareness, autonomy, time limitations and physicians on acute care nurses is required to better support nursing practice and improved patient outcomes. The goal of this study is to provide information that enhances the body of nursing knowledge related to these factors. 


\section{Electronic Health Records and Clinical Decision Support Systems}

Current electronic health records (EHRs) support data entry, however, due to their cumbersome nature, limit data retrieval of important patient information (Carrington \& Effken, 2011). Clinical decision support systems (CDSS) were intended to relieve this frustration for end users using patient data, algorithms, and reference knowledge bases. CDSS use patient data as inputs to activate an algorithm with a reference knowledge base to generate an output, seen by the end user as a clinical alert (Coiera, 2003). Derived from literature, vetted expert opinion, accepted parameters, or the reference knowledge base, the alert is designed to inform decisionmaking (Spooner, 2007).

Despite the advantages of this technology, CDSS have profound limitations towards nurse decision-making. Alert fatigue and the short life-span of reference knowledge threaten the potential positive impact of CDSS towards patient safety. Nurses choose to override clinical alerts or ignore CDSS, demonstrating their lack of relevance in acute care (Spooner, 2007). Another contribution to the poor adoption of CDSS is that they are not designed using a decision-making theory (J. Carrington, personal communication, November 19, 2016). Little is known as to whether a CDSS designed using a decision-making theory would reduce alert fatigue and/or increase effective nurse decision-making and patient safety.

\section{Clinical Events}

Sudden and unexpected changes in patient condition require the nurse to make quick decisions at the point of care. Acute care nursing requires nurses to respond to patient needs that are abrupt and occur in a hospital setting (Venes, 2005). In acute care settings, nurses experience many unexpected circumstances that require them to make decisions. Nurses must make 
decisions associated with changes in patient status. Thus, nursing decision-making during a clinical event is critical to positive patient outcomes. Clinical events provide an important framework for this research in that acute care nurses must make decisions in order to provide ideal care for patients when they experience a clinical event.

Sudden and unexpected changes in patient condition have been conceptualized as Clinical Events (CEs) and may serve as a means to increase our understanding of nurse decisionmaking in acute care. Carrington first described CEs as sudden and unexpected changes in patient condition that could lead to incidences of failure to rescue or unexpected deaths (Carrington, 2008; Carrington \& Effken, 2011). Later, Carrington then defined CEs as sudden and unexpected changes in patient condition represented by fever, bleeding, pain, changes in level of consciousness, respiratory status, and output (Jansen, Surdeanu, Forbes, Carrington, 2013). CEs have yet to be defined by quantitative measure, such as specific body temperature for fever, level of pain using a scale, or type of output experienced as a change in condition ( $\mathrm{J}$. Carrington, personal communication, November 19, 2016). This flexibility in the definition of CEs allows for nurse interpretation during patient assessment and a means for exploring nurse decision-making (J. Carrington, personal communication, November 19, 2016).

\section{Problem Statement}

Therefore, current research suggests that nurse decision-making is influenced by experience, organizational culture factors, education and expertise, situation awareness, confidence, time limits, physician influence, and the decision model used to explore this science. We have yet to understand nurse decision-making processes from the point of care to continuing care for the same patient who experienced a sudden and unexpected change in condition. This 
research builds upon the work of Carrington (2012) and Klein, Calderwood, and Clinton-Cirocco (2010) and seeks to increase our understanding of nurse decision-making and increase patient outcomes by taking the innovative approach of exploring decision making in time limited circumstances. This research also serves as foundational in understanding nurse decision-making towards the development of theory based CDSS for improved technology assisted decisionmaking and increased patient safety.

\section{Purpose of Research}

The purpose of this study is to explore nurses' perceptions of clinical decision-making for a patient who experienced a clinical event.

\section{Research Definitions}

The following definitions will be used for this research:

- Decision making: '...selection of one option from two or more options.' (Klein et al., 2010, p. 186).

- Decision making in nursing: includes recognition of patient problems, determination of a best course of action, with the goal of enhancing patient outcomes continuously throughout hospitalization (Benner, Hughes, \& Sutphen, 2008; Hagbaghery, Salsali, \& Ahmadi, 2004)

- Nurse Experience: is defined as years of experience working as a nurse.

- Responding nurse: Nurse who is caring for the patient at the time of the CE.

- Receiving nurse: Nurse who continues care for the patient who experienced a CE before change of shift. 


\section{Research Questions}

This research will answer the following questions:

1. What processes do nurses describe in decision-making for patients who experience a sudden change in condition?

2. What are the similarities and differences in decision-making processes between responding and receiving nurses?

3. What nurse characteristics influence the decision-making process?

\section{Significance of Research}

Ineffective decision making has been linked with up to 98,000 deaths each year (Kohn, Corrigan, \& Donaldson, 1999). Numerous factors create a complex decision making environment for acute care nurses. Acute care nurses may care for up to five patients at a time in acute care (Tanner, 2006). This challenging patient care environment results in critical care nurses making decisions every 30 seconds (Bucknall, 2000). Due to the considerable potential benefits associated with improving decision making in nursing, research focused on better understanding of decision making in order to support enhanced decision making is essential for improved patient outcomes.

\section{Summary}

This research seeks to expand on the nursing body of knowledge related to nursing decision making in acute care. Time limits, multiple members of the health care team, experience, situation awareness, the decision model used are all factors identified in the literature as important to decision making in acute care. Clearly, additional research is important to 
describe these factors and promote decision making and decision making support in nursing practice. 


\section{CHAPTER II: CONCEPTUAL FRAMEWORK}

This chapter will describe the theoretical framework that will guide this research. This research will build on previous research identifying the 'effective nurse-to-nurse communication framework' described by Carrington (2012). The overarching framework is the Carrington (2012) communication framework. Naturalistic decision-making (NDM) framework will guide this research related to nurse decision-making.

\section{Communication Framework}

The Communication Framework details several aspects important to communication between responding and receiving nurses involved in the care of a patient experiencing a sudden change of condition described as a clinical event (Carrington, 2012a). This framework integrates Symbolic Interaction Theory as described by Mead (1967) and Blumer (1969) and Information

Theory as described by Shannon (1967). Symbolic Interaction theory includes the concepts mind (interpretive process), self (meaning established through individual experiences), and society (social interaction with environmental stimuli) (Blumer, 1969; Mead, 1967). Information theory includes the sender (the constructor of the message), device (tool used for communication), and the receiver (recipient of the sender's message through the device) (Shannon, 1967). Information Theory includes entropy (message uncertainty), negentropy (increased information for message clarity), redundancy (repetition of a message which increases negentropy), noise (factors that increase uncertainty), and probability (of message developed and content of message) (Shannon, 1967). Together these theories define important concepts for the communication of a clinical event. 
Nurse to nurse communication as described by Carrington (2012a), begins with the patient presenting with a sudden change in condition or clinical event. This patient is identified and classified by the responding nurse whose communication is influenced by their characteristics and perceptions. The electronic and verbal communication system in which the nurses and patient interact identifies the means through which this communication occurs. This process continues with the receiving nurse perceiving the communicated message and includes the influences of this nurse's perceptions and characteristics. The Communication Framework describes the communication process between responding and receiving nurses in a manner that allows for better understanding, through research, of this process.

This framework is very effective in describing nurse to nurse communication. The framework also accounts for nurse characteristics and their influence on communication of a clinical event (CE). The ENNC is limited, however, in that it does not account for nurse decision-making when approaching the patient who has experienced a $\mathrm{CE}$ or the receiving nurse continuing care when approaching the patient. Here it is hypothesized that nurses perform decision-making at four stages of care: Responding nurse when they approach the patient and when they communicate the $\mathrm{CE}$ and the Receiving nurse, when they receive the message and again when they approach the patient. To further explore these elements of decision-making and eventually communication, Naturalistic Decision-Making Theory will then guide this research.

\section{Naturalistic Decision-Making Theory}

Historically, decision-making research has focused on many approaches with one of the most researched being Classical Decision Making (CDM) (Cioffi, 2012; Lipshitz, Klein, Orasanu, \& Salas, 2001). CDM includes a choice from options, consideration of the potential 
consequences based on the decision maker's needs, an exploration of all possible options, and a selection of an option that is '...context-free and amenable to quantitative testing' (Lipshitz et al., 2001, p. 333). Traditionally, CDM predominates as the focus of decision making research in nursing (Cioffi, 2012).

NDM is defined as ' ...the way people use their experience to make decisions in field settings' (Zsambok, 1997, p. 4). Research using NDM discovered that expert decision makers do not find the CDM approach helpful when decision making in actual settings that involve specific key factors (Lipshitz et al., 2001). These key factors include '...ill-structured problems (not artificial, well-structured problems), uncertain dynamic environments (not static, simulated situations), shifting, ill-defined goals (not clear and stable goals), action feedback loops (not oneshot decisions), time stress (as opposed to ample time), high stakes (not situations devoid of true consequences for the decision maker), multiple players (as opposed to individual decision making), organizational goals and norms (as opposed to decision making in a vacuum)' (Orasanu \& Connolly, 1993 as cited in Zsambok, 1997, p. 5). In the original NDM research, using Fire Ground Commanders (FCG) as participants, it was determined that expert decision makers do not examine an exhaustive list of decision options when the key factors associated with NDM exist (Klein et al., 2010). Instead, expert decision makers were found to examine only one option at a time during decision-making situations that include the NDM key factors (Klein et al., 2010).

The FCG study implemented an interview guide and asked the experienced (12-37 years' experience) FCG participants to report a non-routine event in detail (Klein et al., 2010). The interviewer then asked more probing questions to further enhance understanding of factors such 
as time line associated with the described event. Results of this research include that the decisions were time pressured (Klein et al., 2010). In addition, out of 156 decision points only 10 were described as including contrasting of options by the decision maker (Klein et al., 2010). Generally, the decisions were made following the decision maker remembering a similar situation from their previous experience and "matching" it rather than incorporating a formal evaluation (Klein et al., 2010, p. 193). This matching led the FCG to develop a course of action based on their previous experience (Klein et al., 2010). This describes 127 of the 156 decision points in this research (Klein et al., 2010). Situation awareness (SA) was found to be important to this process and is defined as: “...identifying and clarifying the current state of the world including goals and assumptions..." (Klein \& Calderwood, 1991, p. 1018), This research also refers to SA as "predecision processes". SA is further described as the "driving factor" in decision-making (Endsley, 1997). SA includes perception (awareness of the setting and circumstances of the situation), comprehension (understanding the meaning of the setting and situation), and projection (a forecasting of what will occur) (Endsley, 1997). Situation awareness is essential for initial recognition of a situation as "typical" and adjusts throughout the decisionmaking process as more information is available to the decision maker (Klein et al., 2010). See Figure 1 for this author's diagram of NDM based on descriptions from the above literature. 


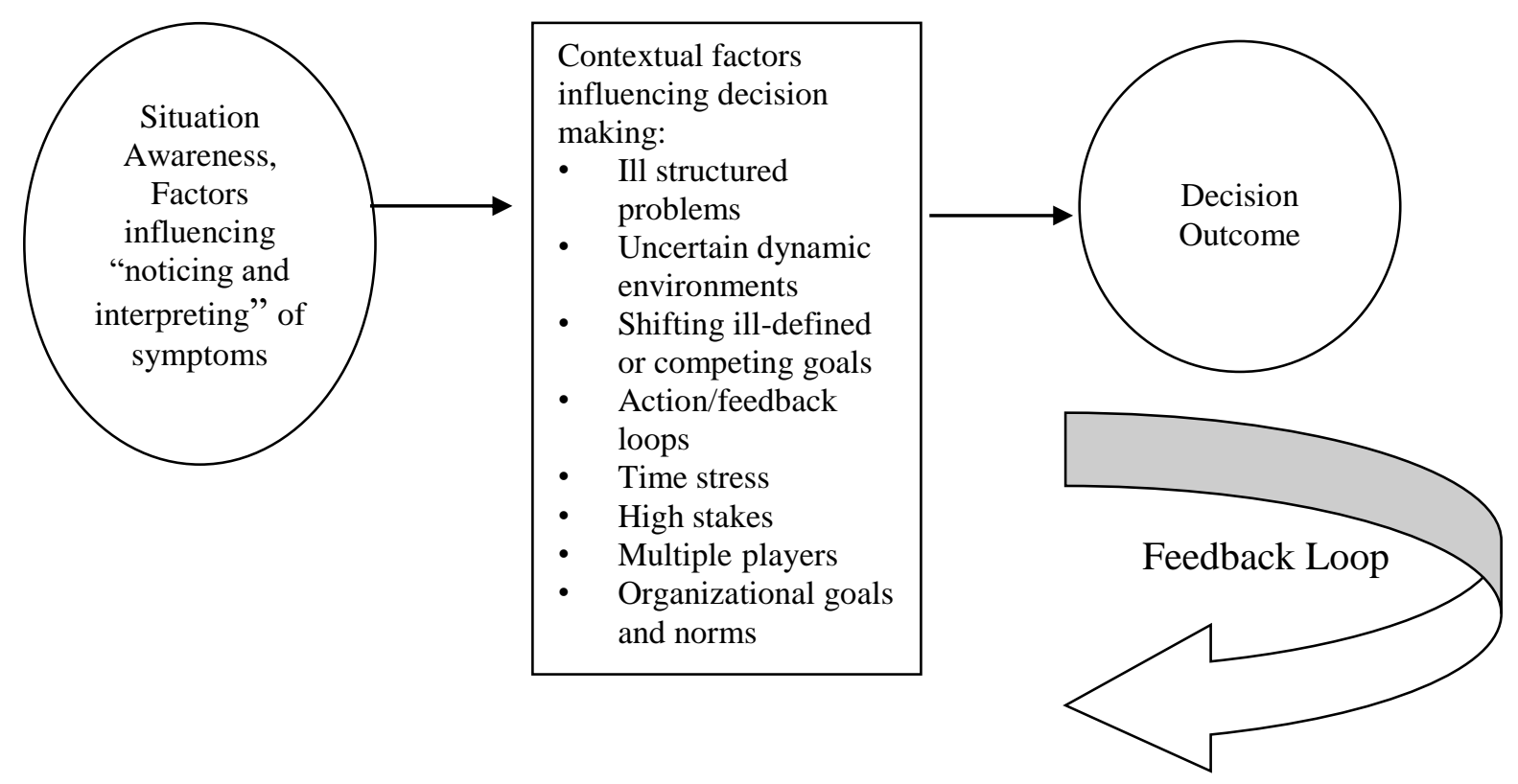

FIGURE 1. Naturalistic Decision Making Based on Literature.

Few examples exist where nurses have used NDM to explore nurse decision-making. A Cumulative Index of Nursing and Allied Health Literature (CINAHL) search using (Major headings (MH) "Nursing Practice") AND naturalistic decision making yielded one result. A CINAHL search using: (MH "Research, Nursing") AND naturalistic decision-making yielded no results. A PubMed search using (nursing) AND naturalistic decision making yielded no results. A gap in nursing research exists because acute care nurses must make decisions that include the key factors of NDM when caring for patients experiencing a clinical event. Therefore, this conceptual framework was determined to be useful to better understand acute care nurses' decision making.

Additional definitions important to NDM include situation awareness, pattern matching and decision point. SA is not specifically defined in the initial FGC study but is described as important in initial decision-making and throughout the decision-making process (Klein et al., 
2010). Pattern matching (prototype identification) is not specifically defined but is referred to in the FGC study: "A fire could be spoken of as typical, which suggested our next category: prototype." And "The FGCs encounter a decision point they recognize a match to a prototype, and the prototypical scenario guided by experience tells them how to proceed" (Klein, Calderwood, \& Clinton-Cirocco, 2010, p. 194). Further discussion states that a "course of action" is used without deliberation over other options. This led to the decision makers not realizing that they had followed a decision-making process. A decision point occurred when the FGCs identified that alternate courses of action could have been used (even if they were not considered during the time as options) (Klein \& Calderwood, 1991). These definitions add to the descriptions of NDM to help with overall understanding of this conceptual framework.

\section{Evaluation of NDM}

Gary Klein is credited as the primary researcher associated with NDM with origins in experimental psychology (Klein et al., 2010). Prior NDM research exploring decision making has focused on fire fighters, warfare technicians, power plant operators, chess players, anesthesiologists, and clinical nurse specialists (Carvalho, dos Santos, \& Vidal, 2005; Hepgul et al., 2012; Klein, Wolf, Militello, \& Zsambok, 1995; Klein et al., 2010; Phipps \& Parker, 2014; Randel, Pugh, \& Reed, 1996). The NDM framework has been tested using numerous professions that share characteristics in decision-making consistent with complex environments and time limitations, such as: warfare technicians (Randel, Pugh, \& Reed, 1996), power plant operators (Carvalho, dos Santos, \& Vidal, 2005), and chess players (Klein, Wolf, Militello, \& Zsambok, 1995). In addition, the key factors are defined clearly stated in non-profession specific terms allowing them to be applied in many professions (See previous discussion on definitions). The 
variety of professions in which NDM has been applied in the past supports the argument that this framework may be useful in nursing.

To determine the strength of NDM for use in research Walker and Avant (2011) will be used as a guideline for evaluation. Please see earlier discussion for the actual definitions. The defined concepts are abstract in that they are not limited to time or space and are not directly measurable (Walker \& Avant, 2011).

NDM research includes situations that fit the defining markers (for instance, chess players find their decisions to be high stakes in a similar manner as power plant operators). The terms are stated in brief non-profession specific terms making them applicable to many decisionmaking settings. Several of the terms important to the framework are not defined specifically in the original FGC study but are implied. The specific definitions were published in other sources later and are congruent with the implied meanings in the original study. The definition of NDM is theoretical in that there are no operational limitations for classifying or measuring (Walker \& Avant, 2011). Relational statements include the defining markers, which are lower order concepts and help to define a situation as NDM. These definitions could also be viewed as descriptive in that specific situations for use and operational measures are not identified (Walker \& Avant, 2011). The defining markers are important in defining a decision-making situation as NDM (Zsambok, 1997). The relationship between the concepts is not causal, associated in a positive or negative manner, or linearly related as defined by Walker and Avant (2011). Situation awareness, pattern matching and decision points are not defined in a causal manner but may be viewed as part of a process based on the description in the FGC study. 
NDM has a broad focus as evidenced by its application to research in many professions (See examples of other studies). This framework predicts that experienced decision makers will make decisions in real-world situations that align with the key factors by developing and maintaining situation awareness, pattern matching (context laden, prototype identified), and using an option that is not selected from a list of options but based on the prototype. The decision maker is often not consciously aware of this as a decision-making process (Klein, Calderwood, \& Clinton-Cirocco, 2010; Zsambok, 1997).

Several studies support the use of NDM in the exploration of decision making in realworld settings. This framework has provided valuable insights into the decision-making processes of experienced decision makers. The traditional (normative) method (involving a formal selection of an alternative from a list of alternatives) that has been generally accepted has not been found to be used in decision making situations specific to NDM (Lipshitz, Klein, Orasanu, \& Salas, 2001). NDM is a useful framework because it further examines decisions made during circumstances that meet defining markers (Walker \& Avant, 2011). The straightforward language used in this framework is non-exclusive to a particular profession. This strengthens its usefulness in other professions. Because this framework has not been widely implemented in nursing research, use of NDM could more fully explain how experienced nurses make decisions in high stakes situations. Results of research using NDM in acute care environments can help to inform further research related to improving decision making in clinical practice, improving nursing education so that nurses are better prepared to make decisions upon graduation, and ultimately improve patient outcomes. 
Because this framework can be widely used it is generalizable (Walker \& Avant, 2011). The abstract qualities of NDM and its non-specific terms enhance the generalizability of this framework to various areas of nursing. This framework is parsimonious because the terms and defining markers of NDM are succinct, simple, and broad in wording (Walker \& Avant, 2011). The simplicity of this framework increases its understandability. While, many professions have found this framework to be helpful in describing decision making by experienced professionals in particular situations this framework is used for description and not for measurement. Thus, this framework is not testable in in terms of nursing interventions or patient outcomes.

\section{NDM for this Research}

NDM was not developed for use in nursing research and examples of research using this conceptual framework in nursing are very limited. This section will describe findings in existing nursing research that share similarities with NDM as well as describe the theory derivation process for NDM's use in this research.

One rationale for using NDM in this research is that statements and categories identified through literature review on decision-making (Chapter 1) share commonalities with the key factors, definitions, and other important elements in NDM. Examples of statements and categories from Chapter 1 that are similar to the factors of NDM from this literature review include experience (Cappelletti et al., 2014; Husted, 2001; Marshall et al., 2013; Rycroft-Malone et al., 2009; Samuriwo \& Dowding, 2014; Seright, 2011;), time limits (Braaten, 2015; Hedberg \& Larsson, 2003; Hendry \& Walker, 2004; Thompson, Dalgleish, et al., 2008), working with team members including physicians (Krairiksh \& Anthony, 2001; Merrick et al., 2014) and nursing colleagues (Offredy et al., 2008; Marshall et al., 2013; Rycroft-Malone et al., 2009; 
Seright, 2011), organizational culture (Braaten, 2015; Jezewski, 1994; Kihlgren et al., 2006; Krairiksh \& Anthony, 2001; Mantzoukas \& Jasper, 2004; Offredy et al., 2008), and situation awareness (Stubbings et al., 2012; Tower et al., 2012). Due to the number of nursing research articles that identify many of the key factors in NDM in nursing practice and the lack of nursing research using NDM as a framework as a guide to identify new information in nursing, the need for research implementing this framework is evident.

As discussed earlier, NDM framework was not developed for nursing and has not been extensively incorporated into nursing science. Due to this, use of NDM for this research requires theory derivation as theory derivation facilitates the implementation of a theory for nursing research (Walker \& Avant, 2011). Application of theory derivation is appropriate when data are minimal and new information is required (Walker \& Avant, 2011). The initial step in theory derivation is understanding of theory development in the area of research (Walker \& Avant, 2011). The predominate theory applied to nursing decision making research is discussed at the beginning of this chapter. Next, previous research associated with the topic of study is helpful for theory derivation (Walker \& Avant, 2011). The current state of the science in nursing decision making is discussed in Chapter 1. A parent theory for derivation must then be identified (Walker \& Avant, 2011). NDM has been identified as the parent theory for this research based on the theory evaluation and the identified research gap discussed in this chapter. Theory derivation requires that essential content within the parent theory that will be useful for the future research to be determined (Walker \& Avant, 2011). Because the NDM concepts are defined in a nonprofession specific manner and are recognizable for nursing decision making, they will be used in this research. Redefinition of NDM concepts for nursing is important to increase applicability 
for nursing research (Walker \& Avant, 2011). Redefining for nursing could include a redefinition of 'organizational goals and norms (as opposed to decision making in a vacuum)' (Zsambok, 1997, p. 5) as: decision making influences such as nurse managers, physicians, and colleagues in acute care. This definition is based on literature review of nurse decision making in acute care (Nibbelink \& Brewer, 2015). Deriving NDM for use in nursing research is clearly applicable to this research.

\section{NDM and Communication Framework for this Research}

For this research, the adaptation of Carrington's (2012a) Effective Communication Framework (ENNC) includes elements of NDM. This adaptation will focus on the responding and receiving nurses' decision making when approaching the patient to provide care. This research will not include a focus on the communication (including verbal and electronic) between nurses nor the development or interpretation of messages between nurses as was described by Carrington (2012a) but will provide an overarching framework for future research. This future research will include the communication process of the responding and receiving nurses when making decisions associated with a patient experiencing a clinical event. Figure 2 demonstrates this adaptation of Carrington's (2012a) ENNC and NDM. The responding nurse decision maker would progress through the process starting with the perception (involving situation awareness) that a patient is experiencing a clinical event, the key factors describing the decision-making circumstance as NDM, and the nurse's previous experience with similar patient problems leading to a decision that is made for the patient experiencing a clinical event (Decision 1 in Figure 2). The receiving nurse would similarly make decisions when assuming care of a patient who experienced a clinical event prior to their shift (Decision 4 in Figure 2). 
The exploration in this study of the decision-making process used by the responding and receiving nurses, that takes place at 1 and 4 in Figure 2, will provide new information for nursing science related to acute care nursing practice.

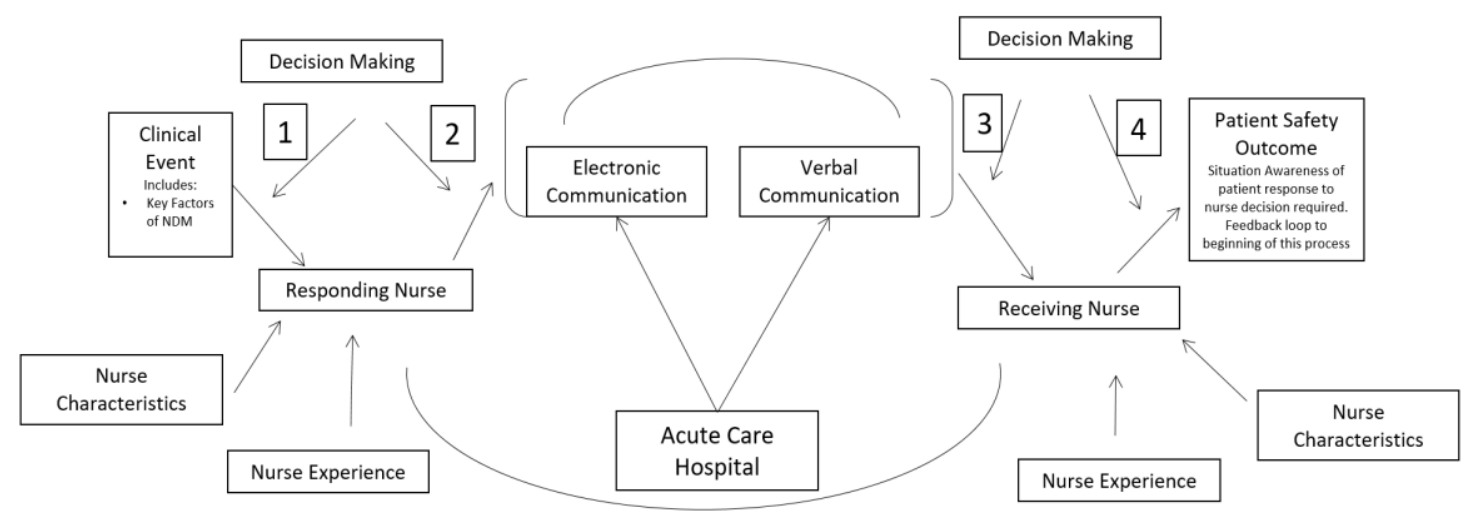

Numbers 1-4 indicate specific decisions that occur in this process

Adapted from Carrington, J. M. (2012). Development of a conceptual framework to guide a program of research exploring nurse-to-nurse communication. CIN: Computers, Informatics, Nursing, 30(6), 293-299

FIGURE 2. ENNC Adapted for this Research

\section{Summary}

The integration of Carrington's ENNC and NDM/RPD will provide a fresh perspective on decision making in acute care nursing. The ENNC provides a guide for continued research to explore decision-making in a novel and innovative manner. Naturalistic Decision-Making Theory serves as a theory to guide our understanding of the processes used by nurses to make decisions for patients who experience clinical events or sudden and unexpected changes.

Specifically, this research will explore and describe decision making factors important to acute 
care nurses in complex circumstances in which the nurse provides care for a patient experiencing a clinical event. 


\section{CHAPTER III: METHODS}

This research used Qualitative Description (QD) as the research methodology. This chapter describes these methodological foci as well as a discussion of trustworthiness and rigor in qualitative research.

\section{Purpose of Research}

The purpose of this study was to explore nurses' perceptions of clinical decision-making for a patient who experienced a clinical event.

\section{Research Questions}

This research sought to answer the following questions:

1. What processes do nurses describe in decision-making for patients who experience a sudden change in condition?

2. What are the similarities and differences in decision-making processes between responding and receiving nurses?

3. What nurse characteristics influence the decision-making process?

\section{Qualitative Research}

Qualitative research is increasingly important to the development of the body of knowledge for nursing (Munhall, 2012). Qualitative research explores and provides information on participants' perspectives of experiences that is of a different theoretical focus than quantitative research through exploration or phenomena that are not reducible to quantifiable measures (Guba \& Lincoln, 1982; Munhall, 2012). This is a constructivist approach as it includes a fresh perspective of the meaning within the data (Crotty, 1998). These perspectives include relevant contextual features identified by the individual as important to the phenomena 
under study that could not be included in a quantitative methodology, which requires control through experimentation (Guba \& Lincoln, 1982). Furthermore, qualitative research acknowledges and values the researcher's influence in the process as opposed to the quantitative approach, which requires the researcher to remain separate and objective (Guba \& Lincoln, 1982). This research seeks to discover new information on the perspectives described by acute care nurses making decisions when caring for a patient experiencing a clinical event. Because this research will explore the perspectives of the participants rather than a measurable outcome, a qualitative method is appropriate for this study.

\section{Qualitative Description}

Qualitative research includes several different approaches. The qualitative method that will be used in this research is qualitative description (QD). QD is a type of qualitative research that includes interpretation but is 'data near' in comparison with other types of qualitative research approaches (Sandelowski, 2010). The interpretation required in qualitative research constructions is influenced by the unique characteristics of the researcher (Sandelowski, 2000). Thus, researchers using QD are unable to completely separate themselves from the data that have been collected (Sandelowski, 2000). QD may include data collection instruments that control the collection of information from participants and may include numbers to assist in summarizing data (Sandelowski, 2000). However, in QD, numbers facilitate data interpretation rather than provide a final analysis of meaning (Sandelowski, 2000). QD is a type of naturalistic inquiry that is based in individual identification of meaning (Lincoln \& Guba, 1985; Sandelowski, 2000). One study employed QD to explore a gap between identified best practice for intravenous device use and actual use of the devices by participants (Downing et al., 2011). This study employed 
interviewing to collect data for theme and category identification (as described by the nurses and clinical assistant participants such as "manual manipulation") and numerical values to describe device preference indicators (Downing et al., 201, p. 293). Use of QD in this study was successful in that the results addressed patient safety issues (Downing et al., 2011). QD provides information that is close to the participants' own words but still provides new meaning through minimal interpretation and has been used to address issues important to nursing practice.

\section{Content Analysis}

Content analysis is appropriate method to analyze data from QD research (Sandelowski, 2000). Content analysis uses a constructivist approach and includes an exploration of emotions and human interactions (Krippendorff, 2003). In addition, qualitative content analysis requires the researcher to interpret the meaning within the data in order to find new meaning (Krippendorff, 2003). However, analysis using QD is discouraged when highly interpretive research is required or when strictly objective quantifiable information is sought (Sandelowski, 2000, 2010). Numbers in content analysis support the meanings found within the data but are not the results of data analysis (Krippendorff, 2003; Sandelowski, 2000). In this research, use of numbers occurred through identification of frequencies in the data to provide a better understanding of the data. Content analysis was determined to best fit the needs for exploring the usefulness of NDM in the beginning understanding of acute care nurses' decision-making when caring for a patient experiencing a CE. QD and content analysis provide essential structure for identification of new meaning within collected data. While QD provides findings that are similar to the collected data, this method requires interpretation and, in this way, provides new meaning for the body of knowledge. 
The approach that was taken for this research was transcription of the audio recorded interviews and the text was analyzed for emerging themes. Themes were organized by subject into categories (Miles et al., 2014). These categories will then be used to answer the research questions.

Analysis, in this research, began concurrently with data collection in that the researcher was responsive to the data as it was collected (Sandelowski, 2010). Memos, notes to provide initial information for category development, and field notes, notes that described factors associated with the environment and the interviews, written during the interview process provided information for initial category development and facilitate understanding through the analysis phase (Lincoln and Guba, 1985; Miles et al., 2014). Audio recorded interviews were downloaded to a secure site and transcribed by a CITI and NIH certified transcriptionist verbatim. Following receipt of the transcribed interviews, the researcher listened to the audio tapes of the interviews and compared them with the transcribed interviews to assure accuracy.

Initial category development included identification of data bits (or statements) within the interviews and involved very little interpretation (Miles et al., 2014). These statements (429 total) were very close to the interviewees' actual words (in most cases, exact quotes) and placed into an Excel spreadsheet. The analysis progressed to identification of themes that emerged among these statements and occurred as more understanding progressed (Miles et al., 2014). These themes were the next phase of table development (in Excel spreadsheets) and included placing the statements that fell within the themes (The themes were named based on interpretation of the meaning within the data bits) into the Excel spreadsheet. The spreadsheets facilitated review and analysis of the data for the author and dissertation chair. The final stage 
was development of categories. Pattern coding then identified relationships among the themes. Themes that were similar in concept were combined into categories (Miles et al., 2014). Some themes were determined to be categories as the data within them were unique and provided important information to answer the research questions. The author developed definitions for the identified themes and categories with an exemplar for each theme and category. The author and dissertation chair discussed the decisions for themes, categories and exemplars and made final definitions. These definitions continually provided a foundation throughout the final stages of analysis to assist in the understanding of themes and categories.

\section{Setting}

The setting for this research was an urban hospital in southern Arizona. Data collection took place in acute care medical and surgical nursing units (including orthopedic/surgical, neurologic, medical/oncologic, and telemetry acute care units) of one community hospital.

\section{Sample}

Ten responding nurses and 10 receiving nurses were recruited from medical surgical nursing units (medical, surgical and telemetry) for a sample of $\mathrm{N}=20$. Purposive sampling was used in order to collect data from participants identified as able to provide "information rich" data to meet the aims of the study (Sandelowski, 2000). Sampling occurred until saturation, or no new concepts emerge, is achieved (Denzin \& Lincoln, 2000). Traditional qualitative research states that recruitment stops when saturation has been reached (Marshall, 1996; Sandelowski, 1995). 


\section{Recruitment}

Following approval from the hospital Institutional Review Board (IRB) where data collection took place, University of Arizona IRB ceded approval to the hospital for data collection (Appendices B and C). This author began data collection using the recruitment flyer and consent approved by the hospital's IRB (Appendices D and E).

Inclusion criteria for this study are as follows: licensed registered nurse $(\mathrm{RN})$, work full time defined as 36 hours per week on their respective unit or as a floating nurse, and cared for a patient who experienced a CE during the shift 24 hours previous to the interview or received hand-off report from a nurse on their unit about a patient who experienced a CE 24 hours previous to the interview. Gender and nursing education level are not criteria for exclusion. Nurses who participate as a responding nurse can also participate as a receiving nurse as the interview questions are different for the two roles (Table 1 of interview questions). None of the participants were interviewed on both roles.

The author met with the Chief Nursing Officer (CNO) of the hospital on the first day of data collection. The $\mathrm{CNO}$ introduced this researcher during a nurse manager meeting. The author described the purpose of this research and described the recruitment, data collection, and consenting processes that would be part of this research. Then the author went to each unit approved for data collection and answered any further questions of nurse managers and charge nurses. Nurse managers were asked to provide information related to nursing staff who could be potential participants in this study. Flyers, approved by the hospital IRB for recruitment, were posted in the nursing break rooms (Appendix D). Staff nurses were approached by this researcher and, if they met inclusion criteria, asked if they would agree to participate in the 
study. The study protocol and consenting process were discussed (Appendix E). These discussions included providing a definition of CEs as described in Chapter 1. Staff nurses were informed of inclusion and exclusion criteria, that the interview and consenting process would take 20-30 minutes in a private location, will not include patient names and that the interview will be digitally audio recorded and will be transcribed for analysis. Participants were informed that there was no right or wrong response to the interview questions and participation would have no impact on their work status and anonymity would be strictly respected. Interviews took place at a time and place within the organization at the preference of the participant, such as a break room. Signed consent forms were stored in a locked file cabinet and data was kept on a password protected laptop. Finally, participants were told that data would be organized using an alphanumeric code. Those nurses who agreed to participate described having cared for a patient who experienced a CE in the previous 24 hours and could be interviewed within the 24 hours of caring for the patient with a CE. Participants were told that they can withdraw from the study without risk or penalty.

\section{Data Collection}

After introduction of the study to the nursing leaders and staff nurses, the occurrence of a $\mathrm{CE}$, and nurse agreement to participate, the consenting process ensued. This research sought to explore nurses' perceptions of clinical decision-making for a patient who experienced a clinical event (CE). CEs were defined for participants as unexpected changes in patient status and include fever, bleeding, pain, change in level of consciousness, change in respiratory status, or change in output (Jansen, Surdeanu, Forbes, \& Carrington, 2013). It was explained to all participating nurses that criteria for participation included having cared for a patient who had 
experienced a CE within 24 hours of the interview. The participating nurse read and signed the consent. The signed copy will be retained by the researcher and placed in a locked box and a copy was provided to the participant for their records.

The interview questions for responding and receiving nurses were not significantly different. The interview employed semi-structured open-ended interview questions. Semistructured open-ended interviewing starts with a list of predeveloped questions which provides a degree of consistency between interviews while also allowing participants to include information that they find important to the subject (Corbin \& Strauss, 2015). Participants were informed that they could choose to not answer a question and that does not result in withdrawal from the study.

After answering participants' questions and consent process concluded, with participants' knowledge, the recorder was turned on and the interview began. Upon conclusion of the interview, the recorder was turned off, participants were given a letter of thanks for their personal file and each participant was given a \$5 Starbucks card (Appendix F). Digitally recorded interviews were then uploaded to a password protected laptop. The recorder and laptop remained in the researcher's possession. The signed consents were locked in the University of Arizona College of Nursing following completion of data collection.

\section{Rigor}

Lincoln and Guba (1985) identify credibility, transferability, dependability, and confirmability as the components necessary for establishing rigor or trustworthiness in qualitative research. Credibility is defined as "truth value" and is identified as the qualitative equivalent of quantitative internal validity (Lincoln \& Guba, 1985). Truth in qualitative research is found in the data (Sandelowski, 1986). Several methods for establishing credibility in research 
have been identified. Prolonged engagement, or spending adequate time with participants will be used to support credibility (Lincoln \& Guba, 1985). In addition to interviewing participants the author spent several hours on the nursing units observing and speaking with potential participants and nursing managers. Triangulation of researchers through inclusion of the dissertation chair and committee in the research process supported a full exploration of the phenomena in this study (Lincoln \& Guba, 1985). Inclusion of outliers or negative case analysis will ensure that all perspectives are included in this research (Lincoln \& Guba, 1985). A variety of experience levels and nursing units were included in the sample that provided a richer collection of data. Referential adequacy is assured through the recording of the interviews to allow for comparison of written findings (Lincoln \& Guba, 1985). Together, these approaches lead to a qualitative research study that is credible.

Next, transferability focuses on whether the findings of the research are applicable for the reader. Transferability can be achieved through a 'thick description' of participants, research setting and other contextual elements that facilitate understanding of the phenomena under study (Lincoln \& Guba, 1985). Transferability relates to quantitative external validity (Lincoln \& Guba, 1985). The reader determines the transferability of qualitative research based on the descriptions provided by the researcher in order to apply it to their circumstances (Lincoln \& Guba, 1985). Transferable research will allow the reader to identify the findings that they are able to apply to their unique circumstance through this researcher's detailed descriptions of the settings and circumstances associated with the data collected.

Dependability is gained through consistency (Lincoln \& Guba, 1985). While quantitative research seeks reliability or replicability, qualitative research seeks to describe only the 
circumstances included in the study (Lincoln \& Guba, 1985). Dependability will be addressed in this study through an inquiry audit. An inquiry audit can be conducted through the use of an auditor (for this research the auditor will be the dissertation chair) who examines the link between the data and the research results to ensure "acceptability" to the auditor (Lincoln \& Guba, 1985, p. 318).

Confirmability is described as the alternate to quantitative objectivity (Lincoln \& Guba, 1985). This research included researcher reflexive journaling, field notes, and an audit trail of the research process to enhance confirmability (Lincoln \& Guba, 1985). An audit trail includes raw data, data reduction products, and notes related to reconstruction for later review (Lincoln \& Guba, 1985). This researcher will store all notes, recordings, and transcripts of this research in a secure but accessible manner for future review to enhance confirmability of the research results.

\section{Data Collection}

Data collection began following human subjects' approval from Northwest Hospital and University of Arizona's Institution Review Board (IRB). The units in which data collection took place were included the following specialties: orthopedic / surgical, neurologic, medical / oncologic, telemetry. During interviews, nurses described caring for between three and six acute care patients on the shift. Each nursing unit included medical technicians as support staff who functioned as nurse assistants and provided assistance to nurses in hands on patient care. The nurses interviewed referred to staff who provided nursing assistance and technical support as "techs". In addition, the nurses on these units used electronic health record (EHR) for charting and were provided with computers on wheels. The EHR provided access to patient medical and nursing records including the medication administration record. 
Nurse Managers on the units included in this study identified nurses who met the study criteria and had cared for a patient experiencing a clinical event or assumed care of a patient who experienced a clinical event within the previous 24 hours. The researcher asked the nurses identified as potential participants for this study, if they would like to participate. If agreed to participate, they were made aware that their participation is voluntary and that they may withdraw at any time without consequence (Corbin \& Strauss, 2015). Participants, nurse managers and the IRBs were made aware that no patient identification information would be sought as part of the interview process and that the focus of this research is to understand nurse decision making not patient specific information (other than asking which CE the patient had experienced). The hospital IRB indicated that this researcher could ask which CE the nurse was reflecting on during the interview but patient information would not be asked. Sampling continued until achievement of saturation, or no new concepts emerge (Denzin \& Lincoln, 2000). The number of participants required to reach saturation can be difficult to predetermine as saturation is identified through incoming data (Corbin \& Strauss, 2015). For this study, saturation was estimated to be reached at 20 participants (J. Carrington, personal communication, October 31, 2016). This researcher requested consent from the Institutional Review Board (IRB) for 20 participants. Data collection ceased after interviews of 10 responding nurses and 10 receiving nurses and saturation was determined to have been achieved.

Data collection methods for this study included semi-structured interview questions. The interview questions are based on the literature and Naturalistic Decision-Making Theory as described by Klein et al. (2010) and listed in Table 1. Literature review provides a basis for development of semi-structure interview questions, collection of demographic information, and a 
source for comparison of data collected (M. Koithan, personal communication, March 21, 2015). Initially, all participants were given a consent to read and sign prior to participating in the interview. Any questions regarding participation were answered and the participants were fully informed that their participation was voluntary and that they may cease participation at any time without fear of consequence (Corbin \& Strauss, 2015). Participants were asked if this interview could be recorded. Refer to Appendix E for the consent form used in this study. Participants were informed that participation would take about 20-30 minutes and involve an interview (J. Carrington, personal communication, October 31, 2016). All participants were offered a $\$ 5.00$ gift certificate to Starbucks and a letter of thanks for participation in the study. The interview took place in a setting that was convenient for the participants including the break room on the nursing unit where they work.

Demographic data collection included number of years worked as an acute care RN, number of years working on their current nursing unit, education, and gender (Klein et al., 2010). Because the hospital IRB committee requested patient specific questions to be limited to only the $\mathrm{CE}$ the patient had experienced, no other patient specific questions were asked. Some participants declined to provide their age. Because experience and not age was described as important to understanding decision making processes in NDM and because some participants' age was not available, age was not included as a demographic in this study (Klein et al., 2010). Data collection in QD is guided by the goal of exploring the "who, what, and where of events or experiences" (Sandelowski, 2000). Question development was driven by this focus and based on previously developed questions for gathering information on decision making processes during NDM circumstances. Question development for data collection included adapting questions 
identified as effective in the gathering of information associated with NDM situations (Klein, Calderwood, \& MacGregor, 1989). See Table 1 and Appendix A for examples of study questions and citations. The interview process time limit of 20-30 minutes and participant responses did not allow for all questions to be included in every interview. Further questions were asked based on data collected with the initial semi-structured questions to provide a full description of the event and experience as needed to meet the aim of the study (Patton, 2002).

Data collection began only after approval the IRBs of the hospital where data were collected and University of Arizona IRB ceded approval to this hospital. Following consent signing, the recorder was turned on and the interview began. Brief note taking or memoing was done during the interviews to aid in early theme development (Corbin \& Strauss, 2015).

Descriptions of observations or field notes were recorded during data collection to help identify underlying meaning in the data during data analysis (Spradley, 1980). In addition, all data were de-identified to protect the privacy of the participants through removal of participant specific information and assignment of codes to each participant known only to the researcher (Charmaz, 2014). 
TABLE 1. Responding and Receiving Nurses Interview Questions Aligned with Research Questions

\begin{tabular}{|c|c|}
\hline Research Questions & Interview Questions \\
\hline Demographic Information & $\begin{array}{l}\text { - Full time on this unit or float RN? } \\
\text { - How many shifts have you cared for the patient prior to the clinical } \\
\text { - Hont? } \\
\text { - How many years of nursing experience do you have? } \\
\text { - How many years/months have you worked on your current unit? } \\
\text { - } \quad \text { Phat is you level of nursing education? }\end{array}$ \\
\hline Opening Questions & $\begin{array}{l}\text { RSRN: } \\
\text { - Tell me about an experience you had when caring for a patient who } \\
\text { experienced a CE? } \\
\text { RCRN: } \\
\text { - Tell me about an experience you have had when caring for a patient } \\
\text { who experienced a CE during the previous shift (after receiving report } \\
\text { from the responding RN) }\end{array}$ \\
\hline
\end{tabular}

RQ 1: What processes do nurses describe in decision-making for patients who experience a sudden change in condition?
RSRN:

- Describe events leading up to the CE.

- What were you seeing, hearing, smelling,...?

- What information did you use in making this decision and how was it obtained?

- What were your specific goals at the time?

- What other courses of action were considered by or available to you?

- How was this option selected/other options rejected? What rule was being followed?

- How much time pressure was involved in making this decision?

- When describing this to the RCRN how did you summarize the situation?

- If a key feature of the situation had been different, what difference would it have made in your decision?

RCRN:

- Describe the report you received from the RSRN.

- What information from the RSRN report helped you make nursing care decisions for this patient?

- How was this option selected/other options rejected? What rule was being followed?

- What were your goals at this time?

- What information did you use to make decisions after encountering the patient?

- What were you seeing, hearing, smelling,... while assessing this patient?

- Imagine you were asked to describe the situation to a relief nurse at this point, how would you summarize the situation?

- If a key feature of the situation had been different, what difference would it have made in your decision? 
TABLE 1 - Continued

\begin{tabular}{l}
\multicolumn{1}{c}{ Research Questions } \\
\hline RQ 2: Are there differences in \\
decision-making processes between \\
responding and receiving nurses?
\end{tabular}

RQ 3: Does the decision-making process differ based on nurse experience?

\section{Interview Questions}

RSRN:

- Describe events leading up to the CE.

- What were you seeing, hearing, smelling,...?

- What information did you use in making this decision and how was it obtained?

- If a key feature of the situation had been different, what difference would it have made in your decision?

RCRN:

- What information from the RSRN report helped you make nursing care decisions for this patient?

- How did you feel about the colleague who gave you report? (trust the report?) Did this make a difference in your decision-making?

- How did your decision-making change from receiving report to after patient assessment?

- What were you seeing, hearing, smelling,... while assessing this patient?

- How was this option selected/other options rejected? What rule was being followed?

- Imagine you were asked to describe the situation to a relief nurse at this point, how would you summarize the situation?

- If a key feature of the situation had been different, what difference would it have made in your decision?

\section{Both RSRN and RCRN:}

- In what ways were you reminded of a previous experience?

- What specific training, experience, or knowledge was necessary or helpful in making this decision?

- If the decision was not the best, what training, knowledge, or information would have helped?

- After this experience what advice would you give to a less experienced nurse? Both RSRN and RCRN:

- How has this experience helped you grow as a nurse?

- What is something that you have not thought before that occurred to you during this interview?

- What else do you think I should know to understand better?

- What would you like to ask me?

Note. $\mathrm{RSRN}=$ Responding nurse; RCRN=Receiving nurse

\section{Data Analysis}

All interviews were transcribed from digital recording to text in Microsoft Word and stored on a password protected laptop to maintain security of data. Each interview was 
alphanumerically coded for data organization. Recorded interviews were transcribed by a CITI and NIH certified transcriptionist verbatim and reviewed by the researcher for accuracy. After transcription, each interview then underwent manual qualitative content analysis.

Qualitative content analysis is the method of data analysis used in QD (Sandelowski, 2010). This method of analysis is rooted in the data (Sandelowski, 2000). Coding is used in this method to analyze, provide structure, and summarize the information found within the data with the result of message recognition, exploration of relationships and processes within the data to find the meaning (Krippendorff, 2003; Sandelowski, 2010). Data analysis, coding, occurs concurrently with data collection and is a progressive process (Glesne, 2011; Sandelowski, 2010). Coding includes a meaningful break down of the data but maintains the relationships within the data (Miles, Huberman, \& Saldana, 2014). Structure is also provided through implementation of coding in data analysis (Miles et al., 2014). Coding begins with little interpretation (Miles et al., 2014). This initial step may include emic codes or themes developed that are close to the language of the participant (Bruno, 2013). As more knowledge is gathered, interpretive coding or category development would begin (Miles et al., 2014). Interpretative coding could use etic codes (or in the words of the researcher) (Bruno, 2013). Pattern coding is the final stage of coding and is used in the identification of relationships between the codes (Miles et al., 2014).

In addition, directed content analysis using an existing theory will provide focus for data analysis in this research (Hsieh \& Shannon, 2005). Using directed content analysis, an existing theory guides interview question development, identification of concepts drawn from the theory in initial steps of coding, identification of new concepts if found within the data, and a 
broadening of the theory based on the new findings (Hsieh \& Shannon, 2005). In this research, the key factors of NDM as described by Klein et al. (2010) provided a basis for interview question development. Interviews included questions identified by Klein et al. (1989) as supporting identification of these key factors within the data and were adapted for this research (See Appendix A for interview questions specific to key factors of NDM). It was determined that category development for this research would not be limited to factors identified in NDM as important to decision-making in order answer the research questions in an exploratory manner and to capture data that includes the overarching communication framework.

For this research, the first two interviews were separately coded by the researcher and dissertation committee chair and then compared for level of agreement (Miles \& Huberman, 1994; Miles, Huberman, \& Saldana, 2014). High agreement was determined using a score of 8590\% using the equation: (number of coding agreements)/(number of coding agreements plus number of coding disagreements) (Miles \& Huberman, 1994; Miles, Huberman, \& Saldana, 2014). This initial agreement was calculated to be $88 \%$. This process was repeated after twothirds of the data analysis process and was found to be 95\% (Miles \& Huberman, 1994; Miles, Huberman, \& Saldana, 2014).

Themes that were similar conceptually were placed under larger categories within Excel spreadsheets and were named to further identify meaning and structure with the data. In addition, to facilitating the display of data, the Excel spreadsheets provided assistance in counting frequencies within each theme and category (see tables in Chapter 4 for frequencies) (Miles et al., 2014). The development of the spreadsheets was iterative as data analysis progressed. The 
spreadsheets were retained for review as needed. See Chapter four for tables that include themes, categories, and exemplar statements.

\section{Summary}

The methods described in this chapter meet with the highest current standards for qualitative research. Through conducting research using these methods, the results of this study will provide new and important information that will add to the body of knowledge related to decision making in acute care nursing. 


\section{CHAPTER IV: RESULTS}

This chapter will present the results of this research study. This description will include participant demographics and the clinical events (CE) the nurses reflected on when answering interview questions. Next, the categories, themes, and participant statements will be described and explored for meaning. Finally, answers to the research questions will be discussed.

\section{Trustworthiness}

Trustworthiness was established in this study through application of methods described in Chapter 2. Credibility, transferability, dependability, and confirmability were the guidelines used during this research (Lincoln \& Guba, 1985).

\section{Demographic Data}

The demographic data for this study are displayed in Table 2. The demographic data table groups participants according to demographic variables. The participants included 12 Associate Degree prepared nurses (ADN), seven Bachelor of Science prepared nurses (BSN) and one Master of Science prepared nurse (MSN). The participants had a broad range of experience. Three nurses were within a year of graduating from their nursing programs and six had ten or more years' experience. Two men and 18 women participated in this study and all but one worked exclusively on the unit where they had cared for the patient with a CE. 
TABLE 2. Demographic Data $(N=20)$

\begin{tabular}{|c|c|c|c|c|c|}
\hline & Male & Female & & & \\
\hline \multirow[t]{3}{*}{ Gender: } & 2 & 18 & & & \\
\hline & $10 \%$ & $90 \%$ & & & \\
\hline & This unit & Float & & & \\
\hline \multirow[t]{3}{*}{ Full time on this unit or float? } & 19 & 1 & & & \\
\hline & $95 \%$ & $5 \%$ & & & \\
\hline & $\begin{array}{c}\text { Diploma } \\
\text { Degree }\end{array}$ & $\begin{array}{l}\text { Associate } \\
\text { Degree }\end{array}$ & $\begin{array}{c}\text { Bachelor } \\
\text { Degree }\end{array}$ & $\begin{array}{c}\text { Master's } \\
\text { Degree }\end{array}$ & $\begin{array}{c}\text { Doctoral } \\
\text { Degree }\end{array}$ \\
\hline \multirow[t]{2}{*}{$\begin{array}{l}\text { What is your highest level of } \\
\text { nursing education? }\end{array}$} & 0 & $\begin{array}{c}12 \\
60 \% \\
\end{array}$ & $\begin{array}{c}7 \\
35 \% \\
\end{array}$ & $\begin{array}{c}1 \\
5 \% \\
\end{array}$ & 0 \\
\hline & 0-1 year & 2-3 years & 4-5 years & 6-9 years & $10+$ years \\
\hline \multirow{3}{*}{$\begin{array}{l}\text { Number of years nursing } \\
\text { experience? }\end{array}$} & 3 & 4 & 2 & 5 & 6 \\
\hline & $15 \%$ & $20 \%$ & $10 \%$ & $25 \%$ & $30 \%$ \\
\hline & 0-1 year & 2-3 years & 4-5 years & 6-9 years & $10+$ years \\
\hline \multirow{3}{*}{$\begin{array}{l}\text { Number of years as acute care } \\
\text { nurse? }\end{array}$} & 3 & 4 & 3 & 5 & 5 \\
\hline & $15 \%$ & $20 \%$ & $15 \%$ & $25 \%$ & $25 \%$ \\
\hline & 0-1 year & 2-3 years & 4-5 years & 6-9 years & $10+$ years \\
\hline \multirow{3}{*}{$\begin{array}{l}\text { Number of years/months on } \\
\text { current unit? }\end{array}$} & 8 & 5 & 2 & 4 & 1 \\
\hline & $40 \%$ & $25 \%$ & $10 \%$ & $20 \%$ & $5 \%$ \\
\hline & 1 & 2 & 3 & 4 or more & \\
\hline \multirow{2}{*}{$\begin{array}{l}\text { \# Shifts caring for patient with } \\
\text { CE? }\end{array}$} & 14 & 4 & 1 & 1 & \\
\hline & $70 \%$ & $20 \%$ & $5 \%$ & $5 \%$ & \\
\hline
\end{tabular}

\section{Clinical Events}

Table 3 presents the CEs that occurred and their frequency. Two nurses described caring for a patient who experienced more than one $\mathrm{CE}$ and was added as an additional $\mathrm{CE}$ occurrence in this table. Of the six CEs described by Jansen (2013) and colleagues, each was represented in the data and is further described below.

Pain was the most frequently occurring CE with 12 occurrences. Nurses described that caring for patients with pain to be intensive in terms of patient need and safety. One nurse described the process when caring for a patient with pain: "chart on the pain, and you chart on the level of pain when you give the medication, and then you go back, and you reassess;" P7, RSRN. In addition, nurses described interventions for pain from non-invasive to medication and implications of these intervention for patient improvement as described by this nurse: "There is 
non-pharmacological. You can use ice or whatever, but her pain is a lot to do with surgical pain, and she hasn't mentioned this, but I want to say this is -- she's had chronic pain, like, past pain, so this pain medicine just -- I don't know if she's had it in the past, but it just wasn't working for her, and so I felt like something stronger."; P4 RCRN. The bigger implications of pain and its impact on patient outcomes was also discussed by this nurse: "We definitely want to fix the pain, because then from pain comes not being able to ambulate, not being able to, you know, do things on your own. "; P 11; RCRN. Pain, like other CEs, are part of the complex nursing care of patients in acute care hospital units and leads to many factors that must be considered and addressed in nursing practice.

Change in respiratory status occurred five times in these data. Nurses described several aspects in decision-making when caring for patients experiencing a serious physical change in condition. Two nurses described the importance of experience in this circumstance, for example while caring for patient experiencing a change in respiratory status "You just have to learn through experience, I don't think anybody can teach you, experience has made the confidence."; P2 RSRN. And: “You learn these things in school, and you go through clinicals, but it's just -really, it's been -- over this year."; P3 RCRN.

Change in output occurred three times in these data. One patient experienced fecal incontinence, one patient had diarrhea, and one patient experienced urinary retention following surgery. Fecal incontinence was determined to be a top priority for the nurse, "Things like that (Feces everywhere), you can't really put off."; P5 RSRN. Another nurse found that she had to explore a patient's condition more deeply based on symptoms associated with diarrhea. "I went in this morning to give her meds, and she was complaining of abdominal pain, lower abdominal 
pain. She is on antibiotics, doxycycline. She denies $C$. diff."; P20; RSRN. This nurse, based on the patient's symptoms, believed that it was important to go beyond what the patient said and wanted to assess further: "If it looked like C. diff -- if it was watery, mucousy -- you know, that's one route -- bloody you know, it's going to change, so. And that's going to change a whole lot the stool is the answer."; P20; RSRN. The nurse caring for a patient with urinary retention described several elements that led to her decision-making. For instance, this nurse discussed the hospital protocol for urinary catheterization but emphasized the assessing and talking with the patient: “Asking him to describe what he's feeling as well. Because a lot of times they do say they have the sensation of needing to go."; P19; RCRN. Changes in output therefore, involved different priorities in decision-making based on environment (result of output), symptoms, and hospital protocol.

Fever and bleeding each occurred once and both occurred with the same patient. This patient also experienced a change in respiratory status, pain, and a change in level of consciousness. This nurses' initial comment was 'It's either life or death with this patient. That's how sick he is. "; P1 RSRN. Because this patient was at the end of life, this nurse described her focus as: "So my goal now is just to make him comfortable."; P1, RSRN.

Of these, CEs change in output and pain each occurred twice in one patient. No other patterns in CE occurrence were identified as occurring together in patients. 
TABLE 3. Clinical Events

\begin{tabular}{lcc}
\hline \multicolumn{1}{c}{ Clinical Event } & Frequency of Occurrence & Percent of Occurrence \\
\hline Fever & 1 & $5 \%$ \\
Bleeding & 1 & $5 \%$ \\
Pain & 12 & $50 \%$ \\
Change in Level of Consciousness & 2 & $10 \%$ \\
Change in Respiratory Status & 5 & $25 \%$ \\
Change in Output & 3 & $15 \%$ \\
\hline \multicolumn{1}{c}{ Total } & 24 & \\
\hline
\end{tabular}

\section{Categories, Subcategories, and Thematic Units}

Interviews were transcribed by a CITI and NIH certified transcriptionist verbatim. The author then listened to all the interview recordings and compared them to the transcribed text for accuracy. Inductive qualitative content analysis began with identification of thematic units as described in Chapter 3. The first two interviews were coded separately by the researcher and dissertation committee chair and then compared for level of agreement (Miles \& Huberman, 1994; Miles et al., 2014). Initial coding agreement $88 \%$. This process was repeated after twothirds of the data analysis process had been completed and was 95\% (Miles \& Huberman, 1994; Miles et al., 2014).

Four hundred and twenty-nine statements (429) emerged from the data and were organized into 11 categories. Categories identified included: Awareness of Patient Status, Nursing Roles outside of specific patient care, Goals, Education / Certification / Hospital Training to support decision-making, Experience and Decision-Making, Time Pressure, Teamwork / Support from Staff, Resources, Following Established Routine. Patient Education, and Consideration of Options to Meet Goals. Tables for each category with associated themes, definitions, and exemplar statements identifying the number of statements per category and the 
number of participants who provided statements for each category will be provided within the discussion of individual categories.

\section{Awareness of Patient Status}

Awareness of Patient Status was defined as: having knowledge, consciousness of patient's condition. This category included eight themes: initial assessment, nursing summary of patient status, patient factors that support decision-making, awareness of change in patient status, waiting for patient status to evolve, identification of increased patient need, nursing report to next shift or physician, and developing a picture of patient's baseline. This category included the largest number of statements (139) and all 20 participants provided information supporting this category.

Initial assessment. This theme is defined as appraisal or evaluation and may include: patient history, early goal development. Many of the statements described an initial overview of how the patient presented and patient responses to nursing assessment questions. Thirty-one statements described factors that they found important to describe in their initial assessment of patients experiencing a $\mathrm{CE}$.

Nursing summary. This theme is defined as brief overview of previous patient information based on assessment and was developed from statements made by nurses that captured the overall patient situation. This theme contained nine statements.

Patient factors that support decision-making. The definition for this theme is patient elements that contribute to a patient care situation. Eighteen statements fell into this theme. 
Awareness of change in patient status. Nurses comments that were part of this theme described being aware of a change in patient condition based on appraisal of current patient status. Twenty statements from nurse participants were included in this theme.

Waiting for patient status to evolve. Statements in this theme included those defined as gradually developing patient status. Ten statements were identified as fitting into this theme.

Identification of increased patient need. This theme included statements that described recognition of patient requirement that includes support in addition to nursing staff. This theme included 21 statements.

Nursing report to next shift or physician. This theme was defined as a statement that arises from nursing assessment and shared with other healthcare professionals. Twenty-four statements were included in this theme.

Develop a picture of a patient's baseline. This theme is defined as elaborate on understanding of patient's standard status. This theme included six statements.

TABLE 4. Awareness of Patient Status Category

\begin{tabular}{lll}
\hline \multicolumn{1}{c}{ Category/Theme } & \multicolumn{1}{c}{ Definition } & Exemplar Statement \\
\hline $\begin{array}{l}\text { Awareness of Patient Status } \\
139 \text { statements } \\
20 \text { participants }\end{array}$ & $\begin{array}{l}\text { having knowledge, consciousness } \\
\text { of patient's condition }\end{array}$ & \\
\hline $\begin{array}{l}\text { Initial assessment } \\
n=31\end{array}$ & $\begin{array}{l}\text { Initial appraisal or evaluation and } \\
\text { may include: patient history, early } \\
\text { goal development }\end{array}$ & $\begin{array}{l}\text { I went in the room. She was } \\
\text { tearful. She was crying. She's } \\
\text { complaining of pain. Ifelt her } \\
\text { abdomen. I asked her where the } \\
\text { pain was; P 12 RCRN }\end{array}$ \\
\hline $\begin{array}{l}\text { Nursing summary of patient status } \\
n=9\end{array}$ & $\begin{array}{l}\text { brief overview of patient } \\
\text { information based on assessment at some times and he'll } \\
\text { talk. He has his wife at bedside } \\
\text { holding his hand; P1 RSRN }\end{array}$ \\
\hline
\end{tabular}


TABLE 4 - Continued

\begin{tabular}{|c|c|c|}
\hline Category/Theme & Definition & Exemplar Statement \\
\hline $\begin{array}{l}\text { Patient factors that support decision- } \\
\text { making } \\
n=18\end{array}$ & $\begin{array}{l}\text { patient elements that contribute to } \\
\text { a patient care situation }\end{array}$ & $\begin{array}{l}\text { He's had a history of drug use, } \\
\text { but in terms of his current pain, } \\
\text { he's not -- it doesn't sound like } \\
\text { he's seeking any narcotics or } \\
\text { anything, because we went } \\
\text { through all the medication, and } \\
\text { he opted for something that is not } \\
\text { necessarily a narcotic, so -- it } \\
\text { helped me to kind of jar -- kind of } \\
\text { see where he was coming from.; } \\
\text { P } 16 \text { RCRN. }\end{array}$ \\
\hline $\begin{array}{l}\text { Awareness of change in patient status } \\
n=20\end{array}$ & $\begin{array}{l}\text { being aware of a change in patient } \\
\text { condition based on appraisal of } \\
\text { patient current patient status }\end{array}$ & $\begin{array}{l}\text { He's changed from when I } \\
\text { initially saw him...I walk in and } \\
\text { now he's, like, working to } \\
\text { breathe; P2 RSRN }\end{array}$ \\
\hline $\begin{array}{l}\text { Waiting for patient status to evolve } \\
n=10\end{array}$ & $\begin{array}{l}\text { gradually developing patient } \\
\text { status }\end{array}$ & $\begin{array}{l}\text { see how things go by the end of } \\
\text { the day -- whether she's better or } \\
\text { worse or the same, you, kind of, } \\
\text { go from there.; P } 20 \text { RSRN }\end{array}$ \\
\hline $\begin{array}{l}\text { Identification of increased patient need } \\
n=21\end{array}$ & $\begin{array}{l}\text { recognition of patient requirement } \\
\text { that includes support in addition } \\
\text { to nursing staff }\end{array}$ & $\begin{array}{l}\text { Kind of sounded kind of icky, } \\
\text { diminished lower, little bit } \\
\text { wheezy, So I called respiratory.; } \\
\text { P3 RCRN }\end{array}$ \\
\hline $\begin{array}{l}\text { Nursing report to next shift or } M D \\
n=24\end{array}$ & $\begin{array}{l}\text { a statement that arises from } \\
\text { nursing assessment }\end{array}$ & $\begin{array}{l}\text { she's had some pain control } \\
\text { issues. I've given her this, and } \\
\text { this medication didn't work so } \\
\text { we're converting over to this } \\
\text {...we're looking at discharge and } \\
\text { getting her on oral pain medicine } \\
\text { that works for her for when she } \\
\text { goes home. Then I talk about her } \\
\text { history and what's going to } \\
\text { happen after discharge; P } 4 \\
\text { RCRN }\end{array}$ \\
\hline $\begin{array}{l}\text { Develop a picture of patient's baseline } \\
n=6\end{array}$ & $\begin{array}{l}\text { elaborate on understanding of } \\
\text { patient's standard status }\end{array}$ & $\begin{array}{l}\text { the first thing I did was I assessed } \\
\text { her. I asked her what her baseline } \\
\text { was just to find out how much of } \\
\text { this was actually really, truly } \\
\text { new, and how much of this was } \\
\text { something that was new going on } \\
\text { with her.; P13 RSRN }\end{array}$ \\
\hline
\end{tabular}

Note. $\mathrm{n}=$ number of statements; RSRN=responding nurse; $\mathrm{RCRN}=$ receiving nurse 


\section{Nursing Roles}

Nursing roles outside of specific patient care were defined as customary additional functions required of a nurse not included in the provision of patient care for the patient who experienced the CE. This main category included six statements.

Factors that conflict with $\mathbf{R N}$ ideal goals. This theme was defined as elements of nursing practice or in nursing practice environment that interfere with highest standards of care and included three statements.

TABLE 5. Nursing Roles Category

\begin{tabular}{|c|c|c|}
\hline Category/Theme & Definition & Exemplar Statement \\
\hline $\begin{array}{l}\text { Nursing Roles outside of } \\
\text { specific patient care } \\
9 \text { statements } \\
4 \text { participants } \\
n=6\end{array}$ & $\begin{array}{l}\text { customary additional functions } \\
\text { required of a nurse not included } \\
\text { in the provision of care for the } \\
\text { patient who experienced the } \mathrm{CE}\end{array}$ & $\begin{array}{l}\text { I have to be available for the rest of the staff if } \\
\text { they need help; } P 1 \text { RSRN }\end{array}$ \\
\hline $\begin{array}{l}\text { Factors that conflict with } R N \\
\text { ideal goals } \\
n=3\end{array}$ & $\begin{array}{l}\text { Elements of nursing practice or } \\
\text { in nursing practice environment } \\
\text { that interfere with highest } \\
\text { standards of care. }\end{array}$ & $\begin{array}{l}\text { So it's just not feasible to do that at night } \\
\text { when we're running on kind of a skeleton } \\
\text { crew. So I had to tell her no, huh, which is } \\
\text { hard, but we did talk about how tomorrow talk } \\
\text { to the therapist and see if that's something you } \\
\text { could do, and we talked about how that would } \\
\text { be good for her lungs; P 8; RCRN }\end{array}$ \\
\hline
\end{tabular}

Note. $\mathrm{n}=$ number of statements; RSRN=responding nurse; RCRN=receiving nurse

\section{Goals}

Goals were described as efforts focused on particular aim or aims. This category included four statements.

Immediate need goals. This theme was defined as efforts toward an aim that cannot be delayed. This theme included 11 statements. 
Goals for the present shift/prior to discharge. The definition for this theme was efforts toward aim that should be met before report to next shift or before patient goes home. There were 13 statements in this theme.

Discharge goals. This theme was defined as efforts toward aim(s) that should be met by the time a patient goes home and may continue to support patient well-being in the home. Three statements were included in this theme.

RN intervention to meet goals. The definition for this theme was nursing actions designed to meet aims. Three statements were identified as fitting into this theme.

TABLE 6. Goals Category

\begin{tabular}{|c|c|c|}
\hline Category/Theme & Definition & Exemplar Statement \\
\hline $\begin{array}{l}\text { Goals } \\
30 \text { statements } \\
8 \text { participants }\end{array}$ & $\begin{array}{l}\text { efforts focused on particular aim or } \\
\text { aims }\end{array}$ & \\
\hline $\begin{array}{l}\text { Immediate need goals } \\
n=11\end{array}$ & $\begin{array}{l}\text { efforts toward aim that cannot be } \\
\text { delayed }\end{array}$ & $\begin{array}{l}\text { We'll have to get that (pain) under } \\
\text { control right then and there.; P4 RCRN }\end{array}$ \\
\hline $\begin{array}{l}\text { Goals for the present shift/prior to } \\
\text { discharge } \\
n=13\end{array}$ & $\begin{array}{l}\text { efforts toward aim that should be } \\
\text { met before report to next shift or } \\
\text { before patient goes home }\end{array}$ & $\begin{array}{l}\text { she needed some testing. They needed } \\
\text { to find out what was going on, but we } \\
\text { want to keep her comfortable until they } \\
\text { can find out what the cause of the pain } \\
\text { is; P 12; RCRN }\end{array}$ \\
\hline $\begin{array}{l}\text { Discharge goals } \\
n=3\end{array}$ & $\begin{array}{l}\text { efforts toward aim(s) that should be } \\
\text { met by the time a patient goes home } \\
\text { and may continue to support patient } \\
\text { well-being in the home }\end{array}$ & $\begin{array}{l}\text { And then also we plan in when he does } \\
\text { discharge, is he going to have any } \\
\text { needs admission to discharge, ... what } \\
\text { support he has at home; P } 2 \text { RSRN }\end{array}$ \\
\hline $\begin{array}{l}R N \text { intervention to meet goals } \\
n=3\end{array}$ & $\begin{array}{l}\text { nursing actions designed to meet } \\
\text { aims }\end{array}$ & $\begin{array}{l}\text { she was anxious and nauseous, so I } \\
\text { gave her the Zofran to help with the } \\
\text { nausea. She had Ativan.; P 20; RSRN }\end{array}$ \\
\hline
\end{tabular}

Note. $\mathrm{n}=$ number of statements; RSRN=responding nurse; RCRN=receiving nurse 


\section{Education/Certification/Hospital Training to Support Decision-Making}

This category was defined as knowledge attained in either a nursing academic setting or in the facility in which patient care takes place. This category had no themes and included 13 statements.

TABLE 7. Education/Certification/Hospital Training to Support Decision-Making Category

\begin{tabular}{|c|c|c|}
\hline Category/Theme & Definition & Exemplar Statement \\
\hline $\begin{array}{l}\text { Education/Certification/Hospital } \\
\text { Training to support decision-making } \\
13 \text { statements } \\
8 \text { participants }\end{array}$ & $\begin{array}{l}\text { knowledge attained in either a } \\
\text { nursing academic setting or in } \\
\text { the facility in which patient } \\
\text { care takes place }\end{array}$ & $\begin{array}{l}\text { I don't know, I go back to a lot of } \\
\text { what I learned in school, and then of } \\
\text { course, being new, I, you know, ask } \\
\text { a lot of questions; P5 RSRN }\end{array}$ \\
\hline
\end{tabular}

Note. $\mathrm{n}=$ number of statements; RSRN=responding nurse; $\mathrm{RCRN}=$ receiving nurse

\section{Experience and Decision-Making}

This category was defined as previous encounters or observations with patient care situations. Themes and exemplar statements are described below.

Link between experience and confidence. Statements that were identified as belonging to this category were described as belief in abilities based on previous encounters. This theme had four statements.

\section{Previous experience with similarities or differences in patients that influence}

decision-making. The definition for this theme was identification of recognition of patient care situation based on previous patient encounters. Eighteen statements were included in this theme.

Experience and education in decision-making. This theme was a description of the interaction between knowledge attained in education environment and patient encounters. This theme included three statements. 
Experience influence on decision-making/nursing practice. The definition for this

theme was previous encounters or observations with patient care situations that effect decision

making. This theme included 40 statements.

\section{Reflecting to when inexperienced/development of confidence through experience.}

Nursing statements in this category included those described by the definition: statement related

to time of inexperience as a registered nurse. Seven statements were included in this theme.

TABLE 8. Experience and Decision-Making Category

\begin{tabular}{|c|c|c|}
\hline Category/Theme & Definition & Exemplar Statement \\
\hline $\begin{array}{l}\text { Experience and Decision-Making } \\
72 \text { statements } \\
18 \text { participants }\end{array}$ & $\begin{array}{l}\text { previous encounters or } \\
\text { observations with patient } \\
\text { care situations }\end{array}$ & \\
\hline $\begin{array}{l}\text { Link between experience and } \\
\text { confidence } \\
n=4\end{array}$ & $\begin{array}{l}\text { belief in abilities based on } \\
\text { previous encounters }\end{array}$ & $\begin{array}{l}\text { Or, you know, to be able to say, hey, I've } \\
\text { never done this before, you know. I'm an } \\
\text { experienced nurse, I've done a lot of things, } \\
\text { but I also have no problem telling doctors } \\
\text { I've never done this or I've never seen this.; } \\
\text { P13; RSRN }\end{array}$ \\
\hline $\begin{array}{l}\text { Previous experience with similarities } \\
\text { or differences in patients that } \\
\text { influence decision-making } \\
n=18\end{array}$ & $\begin{array}{l}\text { identification of } \\
\text { recognition of patient care } \\
\text { situation based on previous } \\
\text { patient encounters }\end{array}$ & $\begin{array}{l}\text { I've seen a lot of patients in this situation so I } \\
\text { knew the signs; PI RSRN }\end{array}$ \\
\hline $\begin{array}{l}\text { Experience and education in } \\
\text { decision-making } \\
n=3\end{array}$ & $\begin{array}{l}\text { description of the } \\
\text { interaction between } \\
\text { knowledge attained in } \\
\text { education environment and } \\
\text { patient encounters }\end{array}$ & $\begin{array}{l}\text { education gives basis, my experiences make } \\
\text { things smoother and guides me with } \\
\text { education, education tells me why we do the } \\
\text { things we do; P } 2 \text { RSRN }\end{array}$ \\
\hline $\begin{array}{l}\text { Experience influence on decision- } \\
\text { making/nursing practice } \\
n=40\end{array}$ & $\begin{array}{l}\text { previous encounters or } \\
\text { observations with patient } \\
\text { care situations that effect } \\
\text { decision making }\end{array}$ & $\begin{array}{l}\text { Really, really, I just say it's experience. I } \\
\text { mean, in the very beginning, like I said, I } \\
\text { would -- I remember freaking out about all } \\
\text { the new, little things that now I'm, like, no; } \\
\text { P6 RSRN }\end{array}$ \\
\hline $\begin{array}{l}\text { Reflecting to when } \\
\text { inexperienced/development of } \\
\text { confidence through experience } \\
n=7\end{array}$ & $\begin{array}{l}\text { statement related to time of } \\
\text { inexperience as a registered } \\
\text { nurse }\end{array}$ & $\begin{array}{l}\text { built my confidence through each. I } \\
\text { remember the first time I called an RRT. So } \\
\text { much crazy. Now, I feel like the chaos has a } \\
\text { rhythm to it; P } 2 \text { RSRN }\end{array}$ \\
\hline
\end{tabular}

Note. $\mathrm{n}=$ number of statements; RSRN=responding nurse; RCRN=receiving nurse 


\section{Time Pressure}

Time pressure was described as feeling compelled to make decisions or achieve aims within a time period. Exemplar statements are provided below to facilitate understanding factors that contribute to time pressure. This main category included 13 statements.

Prioritization. Prioritization was described as identification patient care described as taking precedence. There were 12 statements in this theme.

Delegate. The definition of this theme was identified appointment of another staff member to carry out patient care when necessary due to nurse task load. There were two statements included.

Patient care prioritization based on stability. This theme included statements defined as establishment of patient care precedence based on deterioration or lack of deterioration. This theme included seven statements.

TABLE 9. Time Pressure Category

\begin{tabular}{|c|c|c|}
\hline Category/Theme & Definition & Exemplar Statement \\
\hline $\begin{array}{l}\text { Time Pressure } \\
34 \text { statements } \\
15 \text { participants } \\
n=13\end{array}$ & $\begin{array}{l}\text { feeling compelled to make decisions or } \\
\text { achieve aims within a time period }\end{array}$ & $\begin{array}{l}\text { I was given a sixth patient early this morning, } \\
\text { and I feel, like, when I'm in a room, I do have } \\
\text { to, like, hurry up and make a decision fast } \\
\text { because I have patients trying to get out of bed, } \\
\text { or they -- other patients that have pain issues } \\
\text { that I need to run to, and then I have physical } \\
\text { therapy calling me, I do you have a time } \\
\text { constraint when I'm in a room to make a } \\
\text { decision fast.; P } 4 \text { RCRN }\end{array}$ \\
\hline $\begin{array}{l}\text { Prioritization } \\
n=12\end{array}$ & $\begin{array}{l}\text { identification patient care described as } \\
\text { taking precedence }\end{array}$ & $\begin{array}{l}\text { right in the middle of med pass, it happens -- } \\
\text { everything, it always happens at the worst time, } \\
\text {--and so, I mean, you just have to -- you can } \\
\text { either sit there and reschedule meds or do what } \\
\text { you need to do to -- but, I mean, something like } \\
\text { that has to be attended to right then and there; } \\
\text { P5 RSRN }\end{array}$ \\
\hline
\end{tabular}


TABLE 9 - Continued

\begin{tabular}{lll}
\hline \multicolumn{1}{c}{ Category/Theme } & \multicolumn{1}{c}{ Definition } & \multicolumn{1}{c}{ Exemplar Statement } \\
$\begin{array}{l}\text { Delegate } \\
n=2\end{array}$ & $\begin{array}{l}\text { identify appointment of another staff } \\
\text { member to carry out patient care when } \\
\text { necessary due to nurse task load }\end{array}$ & $\begin{array}{l}\text { Have to look at which one needs to be seen first } \\
\text { and maybe delegate to other nurses; P1 RSRN }\end{array}$ \\
\hline $\begin{array}{l}\text { Patient care } \\
\text { prioritization based on } \\
\text { stability } \\
n=7\end{array}$ & $\begin{array}{l}\text { establishment of patient care } \\
\text { precedence based on deterioration or } \\
\text { lack of deterioration }\end{array}$ & $\begin{array}{l}\text { If they're in pain or if their vitals are unstable, } \\
\text { I always go to them first. If it's a family } \\
\text { member, they're kind of last on the list.; P10 } \\
\text { RCRN }\end{array}$ \\
\hline
\end{tabular}

Note. $\mathrm{n}=$ number of statements; RSRN=responding nurse; RCRN=receiving nurse

\section{Teamwork/Support from Staff}

This category was defined as coordination of effort of patient care staff within a unit.

There were three themes within this category.

Teamwork/support from staff for decision-making. The definition of this theme was coordination of nursing staff toward making patient care choices. There were 18 statements in this category.

Support from staff to complete patient care. This theme was defined as coordination of nursing staff toward meeting patient care needs. There were seven statements that fit this definition.

Additional support options considered to meet goals. The definition of this theme was coordination of effort of healthcare professionals outside of unit staff to meet patient needs. There were nine statements that were included in this theme. 
TABLE 10. Teamwork/Support from Staff Category

\begin{tabular}{|c|c|c|}
\hline Category/Theme & Definition & Exemplar Statement \\
\hline $\begin{array}{l}\text { Teamwork/Support from } \\
\text { Staff } \\
34 \text { statements } \\
10 \text { participants }\end{array}$ & $\begin{array}{l}\text { coordination of effort of } \\
\text { patient care staff within a } \\
\text { unit }\end{array}$ & \\
\hline $\begin{array}{l}\text { Teamwork/Support from } \\
\text { staff for decision-making } \\
n=18\end{array}$ & $\begin{array}{l}\text { coordination of nursing staff } \\
\text { toward making patient care } \\
\text { choices }\end{array}$ & $\begin{array}{l}\text { I think as nurses, even experienced nurses, we really } \\
\text { like to have that positive feedback, like, this is what } \\
\text { I'm thinking is going on. This is what I'm thinking of } \\
\text { doing. You know, what do you think; P6 RSRN }\end{array}$ \\
\hline $\begin{array}{l}\text { Support from staff to } \\
\text { complete patient care } \\
n=7\end{array}$ & $\begin{array}{l}\text { coordination of nursing staff } \\
\text { toward meeting patient care } \\
\text { needs }\end{array}$ & $\begin{array}{l}\text { I usually try to get the tech first because nurses, you } \\
\text { know, they're busy passing meds, and usually we do } \\
\text { have a tech.; P5 RSRN }\end{array}$ \\
\hline $\begin{array}{l}\text { Additional support options } \\
\text { considered to meet goals } \\
n=9\end{array}$ & $\begin{array}{l}\text { coordination of effort of } \\
\text { healthcare professionals } \\
\text { outside of unit staff to meet } \\
\text { patient needs }\end{array}$ & $\begin{array}{l}\text { can never get in trouble for calling it }(R R T) . . \text { I feel } \\
\text { you always have their support; } P 2 R S R N\end{array}$ \\
\hline
\end{tabular}

Note. $\mathrm{n}=$ number of statements; RSRN=responding nurse; RCRN=receiving nurse

\section{Resources}

The definition of this category was non-nursing sources of support identified as available for aiding in patient care decision making. Fourteen statements were found to be part of this category.

TABLE 11. Resources Category

\begin{tabular}{lll}
\hline Category/Theme & \multicolumn{1}{c}{ Definition } & \multicolumn{1}{c}{ Exemplar Statement } \\
\hline $\begin{array}{l}\text { Resources } \\
14 \text { statements }\end{array}$ & $\begin{array}{l}\text { non-nursing sources of support } \\
\text { identified as available for aiding } \\
\text { in patient care decision making }\end{array}$ & $\begin{array}{l}\text { In the record you can look and see if they have pain } \\
\text { medication order, if it's something that has been going } \\
\text { on since they've been here, how many times they've } \\
\text { needed it; P 17; RCRN }\end{array}$ \\
\hline
\end{tabular}

Note. $\mathrm{n}=$ number of statements; RSRN=responding nurse; RCRN=receiving nurse 


\section{Following Established Routine Category}

The definition of this category was descriptions of customary procedures in patient care (developed either by the individual or by the facility). Fifty statements were found to fit within this category.

Describe viewing patient as individual routinely. The definition for this theme was identified customary approaches designed to identify the needs of the patient as a distinct entity.

Twelve statements were included in this theme.

TABLE 12. Following Established Routine Category

\begin{tabular}{lll}
\hline \multicolumn{1}{c}{ Category/Theme } & \multicolumn{1}{c}{ Definition } & \multicolumn{1}{c}{ Exemplar Statement } \\
\hline $\begin{array}{l}\text { Following Established } \\
\text { Routine } \\
62 \text { statements }\end{array}$ & $\begin{array}{l}\text { Descriptions of customary } \\
\text { procedures in patient care } \\
\text { (developed either by the individual } \\
\text { or by the facility) }\end{array}$ & $\begin{array}{l}\text { first thing I always think about is, like, why are } \\
\text { they here? I go by the diagnosis. Why are they } \\
\text { here, what will that affect, you know, every } \\
\text { other body system; P 11; RCRN }\end{array}$ \\
\hline $\begin{array}{l}\text { Describe viewing patient } \\
\text { as individual routinely } \\
n=12\end{array}$ & $\begin{array}{l}\text { identified customary approaches } \\
\text { designed to identify the needs of the } \\
\text { patient as a distinct entity }\end{array}$ & $\begin{array}{l}\text { There's not usually just one best way especially } \\
\text { when dealing with pain because pain is what } \\
\text { the patient says it is; } P \text { 17; RCRN }\end{array}$ \\
\hline
\end{tabular}

Note. $\mathrm{n}=$ number of statements; RSRN=responding nurse; RCRN=receiving nurse

\section{Patient Education}

Patient education was defined as supporting patient knowledge attainment to enhance achievement of aims. This category included 13 statements.

TABLE 13. Patient Education Category

\begin{tabular}{lll}
\hline Category/Theme & \multicolumn{1}{c}{ Definition } & \multicolumn{1}{c}{ Exemplar Statement } \\
\hline $\begin{array}{l}\text { Patient Education } \\
13 \text { statements }\end{array}$ & $\begin{array}{l}\text { supporting patient } \\
\text { knowledge attainment } \\
\text { to enhance }\end{array}$ & $\begin{array}{l}\text { So I explained to the patient what that was so he can help me if he } \\
\text { experiences the symptoms of compartment syndrome and really }\end{array}$ \\
& $\begin{array}{l}\text { went through the plan. We'll try as much as we could to keep him } \\
\text { pain free, and I made it clear if the medication didn't work, I'd } \\
\text { achievement of aims }\end{array}$ & $\begin{array}{l}\text { reassess the pain after an hour. He was comfortable at that time; } P \\
16 ; R C R N\end{array}$
\end{tabular}

Note. $\mathrm{n}=$ number of statements; RSRN=responding nurse; $\mathrm{RCRN}=$ receiving nurse 


\section{Consideration of Options to Meet Goals}

This category was defined as careful thought of choices for achievement of aims. Nine statements were identified as part of this category.

TABLE 14. Consideration of Options to Meet Goals Category

\begin{tabular}{lll}
\hline \multicolumn{1}{c}{ Category/Theme } & \multicolumn{1}{c}{ Definition } & \multicolumn{1}{c}{ Exemplar Statement } \\
\hline $\begin{array}{l}\text { Consideration of } \\
\text { Options to Meet Goals } \\
9 \text { statements } \\
6 \text { participants }\end{array}$ & $\begin{array}{l}\text { careful thought of } \\
\text { choices for achievement } \\
\text { of aims }\end{array}$ & $\begin{array}{l}\text { If somebody is in pain, making sure you're taking more } \\
\text { than one approach to the issue and -- figuring out what } \\
\text { works best out of all of the things that you could be doing. } \\
; P 17 ; R C R N\end{array}$ \\
\hline
\end{tabular}

Note. $\mathrm{n}=$ number of statements; RSRN=responding nurse; RCRN=receiving nurse

Eleven categories emerged as a result of this research. These categories and themes provide the basis for answering the research questions in this study. Decision-making for acute care nurses in this study was clearly complex for the participants of this research. Numerous elements for a wide variety of CEs were considered by these nurses when they provided care for patients experiencing a sudden change in condition. Each patient situation was unique and while categories emerged in the data, nurses also brought their unique skill set to their nursing practice leading to a broad range of categories.

\section{Answering the Research Questions}

\section{Research Question 1}

The first research question was: What processes do nurses describe in decision-making for patients who experience a sudden change in condition? Processes are stepwise approaches to accomplishing a task. Using content analysis, decision-making elements emerged. These elements were then organized into two broad categories: nurse and organization. The elements are presented below. Processes of decision-making within these data, themes and categories did 
seem to emerge such as consideration of options. However, it was determined that content analysis was not the appropriate method to fully explore the processes and this research focused on the elements described by the participants. The categories and themes could be further divided into two broad elements, the nurse and second, the organization. One category included both nurse and organization broad elements.

The nurse broad element. Two hundred and sixty-three statements make up this broad decision-making element. The decision-making elements related to the nurse include: Awareness of the Patient Status, Nursing Roles, Goals, and Education/Certification/Hospital training to support decision-making, and Experience and Decision-Making. The categories and themes in these elements are as follows.

The Awareness of Patient Status category contained $32 \%$ of the statements that emerged from this research. Within this category, statements included assessment of the patient. Nurses described their overall impression of the patient as well as more specific methods of gathering information. For instance, 15 statements included nursing report. Nurses described asking the patient questions in during assessment in seven statements. Eight statements provided information about the inclusion of patient history in their assessment.

In the Nursing Roles category, one nurse included four statements related to caring for the family. Nurses also discussed the patient load as a factor in caring for a patient experiencing a CE two times.

Nurses' descriptions of Goals, with the exception of three statements that described nursing interventions, indicated that time lines related to goals are important. When patients' 
needs were more critical, the nurses felt a more immediate need for goal attainment. This progressed through goals for discharge which may include patient needs at home.

Nurses described Education/Certification/Hospital Training as an element in decisionmaking. Six education statements describe hospital or education with certification as helpful to nursing practice. Two nurses who discussed their nursing school education indicated that it did not adequately prepare them to be acute care nurses. Three statements indicated that nursing school had been helpful to their current nursing practice.

The influence of Experience on decision-making was described by nurses. Nurses described the importance of previous patient care as the basis for current nursing practice. Included in this is the benefit of having been exposed to patients who were similar to the patient with the CE for which they were currently caring.

The organization broad element. Along with the above nursing elements to decisionmaking, nurses described organizational elements: Time Pressure, Teamwork/Support from Staff, Resources, Patient Education, and Consideration of Options will be discussed below. Nurses described patient load and patient stability as being important factors related to Time Pressure. Nurses prioritized to help them manage the time pressure. This included decisions to not participate in mock codes, delay care for less critical patients and fast decisionmaking.

Nurses described the importance of Teamwork/Support in caring for patients who experienced CEs. Charge nurses were described as highly supportive 12 times in teamwork statements. Rapid response teams were discussed four times as an important source of support in nursing practice. 
The Resource category most often identified, seven times, patient history or medication administration record in the electronic health record as important for nursing decision-making. One nurse described a "pop-up" as part of care for a patient returning to the unit following surgery. This was a clinical reminder for patient care. No other clinical decision support systems in the electronic health record were described as helpful decision-making. Nurses described protocols as important in three statements.

Nurses used Patient Education to educate about voiding following surgery six times, in follow-up for new symptoms three times, and about pain two times. Discussion of the floor routine was the basis of patient education once.

Consideration of other options focused on caring for patients in pain six times. Calling respiratory therapy was discussed once. Options before straight catheterization were also discussed once.

Following Established Routine included elements both Nurse and Organization. Nurses described following routines in their nursing practice that were determined by the organization or personally developed. This category included statements in which the nurse indicated that they approached all patients or patients in a particular circumstance in a customary manner. Pain was discussed 23 times within this category.

This research suggests that acute care nurses include both nurse and organization elements in decision-making while caring for patients experiencing a CE. Nurses include awareness of the patient, roles, goals, education and experience as influential in decision-making. Beyond these, nurses also acknowledged elements that are result of organizational decisionmaking: time pressure, teamwork, resources, and patient education. 


\section{Research Question 2}

The second research question posed to be answered with this project was: What are the similarities and differences in decision-making processes between responding and receiving nurses? The Effective Nurse-to-Nurse Communication Framework (ENNC) describes important factors to be considered in the communication between nurses who responded to patients experiencing a CE (RSRN) and nurses receiving report (RCRN) on patients who have experienced a clinical event (CE). This research interviewed ten RSRNs and ten RCRNs to explore further the differences between these two groups when making decision for patients experiencing CEs.

RSRN participants had 239 statements and RCRNs had 190 statements. See Table 15 for numbers of statements from each group per category. Overall RSRNs provided 49 more statements than RCRNs when responding to interview questions designed to elicit information related to decision-making in acute care nursing practice. Areas that were particularly high for RSRNs in comparison to RCRNs include awareness of patient status, teamwork, and time pressure.

An RSRN described awareness of patient status as important 77 times. The nurses would describe initial patient interactions for example: "We just can't get him to lower his respiratory rate."; P2 RSRN and other assessment factors that were determined to be important "Heart rate going down quickly."; P1 RSRN. RCRNs provided 62 statements for this category. An RCRN comment included the importance of patient history when making decisions "What's going on with the patient and their history, yeah, it makes a difference." P4 RCRN. Report was not always described as essential: "There wasn't really much report because I already knew her."; P10 
RCRN. However, report did hold important information for an RCRN: "If she was taking two pills, and then overnight she was just taking one. So that helps you kind of have a visual of how it's going to be during the day."; P 11; RCRN.

Nursing roles included care of family for an RSRN: "I'm also making sure she's doing OK too because they're both elderly."; P1 RSRN. An RCRN described nursing practice challenges on night shift: "So it's just not feasible to do that at night when we're running on kind of a skeleton crew. So I had to tell her no, huh, which is hard, but we did talk about how tomorrow talk to the therapist and see if that's something you could do, and we talked about how that would be good for her lungs."; P 8; RCRN.

RSRNs also stated goals as essential to decision-making. Goals included using vital signs as a measure of patient status for this groups of nurses: "I can't leave him with a respiratory of 30, 35, I saw he was fidgety, so I asked if could just put his hands on the side. Respiratory dropped. Why is respiratory going from 17 to 35 with minimal movement."; P2 RSRN. Pain management was important to this RCRN "I wanted to give the Dilaudid in order to just take the edge off so that maybe that oral pain medicine could work."; P 8; RCRN

Education/Certification/Hospital training to support decision-making was described as important to RSRNs. One RSRN discussed three types of certification with one example being: “...certification for nursing practice decisions.”; P1 RSRN. An RCRN did not feel that formal nursing education was what helped her decision-making most: "You learn these things in school, and you go through clinicals, but it's just -- really, it's been -- over this year."; P3 RCRN.

An RSRN found experience to be essential to decision-making: "You just have to learn through experience, I don't think anybody can teach you, experience has made the confidence."; 
P2 RSRN. An RCRN also described the benefit of experience: "I just have had very similar patients. It helps you anticipate what's going to happen next."; P3 RCRN.

An RSRN describe patient factors that help alleviate time pressure. "He seemed stable at the time, and if he says this is an issue that's been going on, then -- and his color, his pulses, his vitals are all good, then I'm not too time crunched."; P9; RSRN. An RCRN describes circumstances that do increase time pressure "You've got five all wanting you, it's kind of, like, who do I see first, and who's more pressing at this point?"; P10 RCRN.

A team approach was described as important by this RSRN: "I think as nurses, even experienced nurses, we really like to have that positive feedback, like, this is what I'm thinking is going on. This is what I'm thinking of doing. You know, what do you think?"; P6 RSRN. An RCRN was appreciative of the support charge nurses provide: "Always call the charge nurse. I'm, like, hey, you know what I mean, this is my issue. Like, what do you think?"; P 11; RCRN.

Resources for one RSRN included information found in the electronic health record: "Well, I guess, I mean, I did think, OK, well, what has she had medication-wise, if anything's been different. So I did look in the MAR to see."; P6 RSRN. An RCRN seemed to describe nursing policies as helpful in only limited situations: “... and even sometimes -- in certain situations, even the policies --for certain things."; P3 RCRN.

When following routine many nurses described pain protocols. For instance, one RSRN stated: "Chart on the pain, and you chart on the level of pain when you give the medication, and then you go back, and you reassess."; P7, RSRN. An RCRN described caring for a patient in pain: "And, I mean, the pain is what the patient says it is, so it was just a 10."; P 11; RCRN 
Patient education was described by an RSRN: "You know, that's something I noticed, anxiety. The more you explain, the more education you give, it seems to make them feel a lot better, knowing a lot of the people say, oh, if somebody would have just told me that, I wouldn't feel like this."; P20; RSRN. An RCRN described her patient education in this manner: "So I explained to the patient what that was so he can help me if he experiences the symptoms of compartment syndrome and really went through the plan. We'll try as much as we could to keep him pain free, and I made it clear if the medication didn't work, I'd reassess the pain after an hour. He was comfortable at that time."; P 16; RCRN

No RSRNs described consideration of options to meet goals. An RCRN described her efforts to provide care for a patient in pain: "There is non-pharmacological. You can use ice or whatever, but her pain is a lot to do with surgical pain, and she hasn't mentioned this, but I want to say this is -- she's had chronic pain, like, past pain, so this pain medicine just -- I don't know if she's had it in the past, but it just wasn't working for her, and so I felt like something stronger. ”; P4 RCRN.

Table 15 lists the roles, and frequencies of emergence in the data. The columns include the categories and numbers of statements provided by RSRNs and RCRNs. Frequencies separated by five or more statements were deemed either RSRN or RCRN. Frequencies separated by less than five points were determined to be shared elements of decision-making by both RSRNs and RCRNs. Differences between RSRNs and RCRNs determined by this method indicate that RSRNs focused on awareness of patient status, nursing roles outside of specific care, education, experience, time pressure, teamwork and support, and resources. In comparison, 
RCRNs prioritized goals, patient education, and options to meet goals of care. Both nursing roles shared following established routine.

TABLE 15. Comparison of Responding and Receiving Nurses and Decision-Making

\begin{tabular}{lcc}
\hline \multicolumn{1}{c}{ Category } & RSRN & RCRN \\
\hline Awareness of patient status & 77 & 62 \\
Nursing roles outside of specific patient care & 8 & 1 \\
Goals & 12 & 18 \\
Education/certification/hospital training to support decision-making & 9 & 4 \\
Experience and decision-making & 40 & 32 \\
Time pressure & 22 & 12 \\
Teamwork/support from staff & 27 & 7 \\
Resources & 11 & 3 \\
Following established routine & 29 & 33 \\
Patient Education & 4 & 9 \\
Consideration of options to meet goals & 0 & 9 \\
\hline \multicolumn{2}{c}{ Total } & 239 \\
\hline
\end{tabular}

Note. Number of statements per category from RSRN (responding nurse) RCRN (receiving nurse)

\section{Research Question 3}

The third research question was: What nurse characteristics influence the decisionmaking process? Nursing research indicates that experience heavily influences the approach to decision-making (Benner \& Tanner, 1987). According to nursing research, novice (time experience) nurses differ greatly from experienced nurses in decision-making. Novice nurses use rules to guide their decision-making while experienced nurses rely on a vast background of patient care situations to support their decision-making (Benner \& Tanner, 1987). The experienced decision maker is described in research as using recognition of patterns and similar situations with intuition to guide their decision making (Benner \& Tanner, 1987). Based on this focus in nursing research on experience and decision-making, an examination of new graduate participants and participants with 10 or more years of experience will be described here. 
This study included three new graduate nurses and six nurses with 10 or more years of experience. Two new graduate nurses had Associates degrees and one had a Bachelor's degree. Two of the more experienced nurses had Associate degrees and four had Bachelor's degrees. One new graduate had seven months experience, one had 11 months experience, and one had one year of experience. To better understand the influence of experience in acute care nursing decision-making, these two groups of decision makers will be compared. Not all categories were represented in the responses from these two groups. The number of responses by each group per category is listed in Table 16 below.

Categories represented among the new graduate nurses in this study differed considerably. The 10 or more years experienced nurses described an awareness of patient status during the interviews seven to 13 times with the exception of one nurse who provided two statements to this category. The less experienced new graduate nurse described the importance of experience and following established routine at a higher rate than the other new graduates which was similar to the highly-experienced nurses. The Bachelor's prepared new graduate did not provide statements to the experience category. The ten or more years' experienced nurses' responses fell into a large variety of categories possibly indicating a great deal of variance in their application of experience in their nursing practice. Other than awareness of patient status, the high scoring categories, experience and following established routine, were largely represented by a relatively few number of inexperienced nurses. The bachelors prepared new graduate nurse did contribute six statements out of 14 to the patient education category. 
TABLE 16. Novice and Experienced Nurse Responses

\begin{tabular}{lcc}
\hline \multicolumn{1}{c}{ Categories Described } & Novice & Experienced \\
\hline Awareness of Patient Status & 11 & 34 \\
Nursing Roles & 0 & 2 \\
Goals & 5 & 6 \\
Education & 3 & 2 \\
Experience & 7 & 17 \\
Time Pressure & 2 & 4 \\
Teamwork & 5 & 0 \\
Resources & 0 & 3 \\
Following Established Routine & 4 & 16 \\
Patient Education & 0 & 3 \\
Consideration of Options & 2 & 0 \\
\hline
\end{tabular}

Note. Number of statements per category from Novice and Experienced participants.

In summary, data were organized to answer three research questions. The first question sought to reveal the process used by nurses to make decisions when caring for a patient who experienced a clinical event. Data analysis associated with answering this question lead to the emergence of categories Awareness of Patient Status, Nursing Roles outside of specific patient care, Goals, Education/Certification/Hospital Training to support decision-making, Experience and Decision-Making, Time Pressure, Teamwork/Support from Staff, Resources, Following Established Routine. Patient Education, and Consideration of Options to Meet Goals. These categories provide new information related to nurse and organizational elements that were described as important for decision-making in the acute care environment. The second research question explored differences between the responding and receiving roles in nursing indicate that communication in the acute care environment leads different approaches in nursing practice. The third research question explored differences in responses contributed by novice and experienced nurses based on the number of years of experience and differing numbers of response to categories. This indicated that years of experience may lead to difference on what nurses view as important to decision-making. 


\section{CHAPTER V: DISCUSSION AND IMPLICATIONS}

This chapter discusses how the findings of this research relate to literature on decisionmaking and nursing practice. In addition, the conceptual frameworks will be discussed based on results from data analysis, the strengths and limitations of this research will be described and future research indicated by the results of this study will be described.

\section{Discussion}

\section{Categories, Themes, and Statements}

The categories that emerged in this research included: Awareness of Patient Status, Nursing Roles outside of specific patient care, Goals, Education/Certification/Hospital Training to support decision-making, Experience and Decision-Making, Time Pressure, Teamwork/Support from Staff, Resources, Following Established Routine. Patient Education, and Consideration of Options to Meet Goals. Most categories were divided into themes based on the statements identified in the data. The research proposal sought to identify processes within the data that guide acute care nurses' decision-making when caring for patients experiencing a clinical event. It was determined that identification of the processes involved in decision-making would require further in depth analysis including possibly a grounded theory approach. This research describes elements identified by acute care nurses as important when caring for a patient experiencing a $\mathrm{CE}$.

Literature review related to decision-making and nursing discussed in chapter 1 reveals a broad array of information. Several factors that emerged as important in this review were found in this research also. 
For instance, several studies found experienced nurses to be important support for nurses making decisions in acute care (Offredy, Kendall, \& Goodman, 2008; Marshall et al., 2013; Rycroft-Malone et al., 2009; \& Seright, 2011). Several nurses in this study discussed reliance on charge nurses if confronted with a challenging patient care decision. The nurses in this study did not specifically state that they sought experienced nurses but the charge nurse role is generally linked with a higher level of experience. Protocols were identified as less helpful than experienced nurses in the literature review (Cappelletti et al., 2014; Husted, 2001; Marshall, West, \& Aitken, 2013; Rycroft-Malone et al., 2009; Samuriwo \& Dowding, 2014; Seright, 2011). Nurses in this study identified usefulness of protocols in five statements out of 429 indicating a lower reliance on protocols' helpfulness in acute care nursing practice.

Nursing unit/organization culture was determined to be important to nursing practice in literature review (Braaten, 2015; Cappelletti et al., 2014; Hendry \& Walker, 2004; Jezewski, 1994; Kihlgren, Forslund, \& Fagerberg, 2006; Krairiksh \& Anthony, 2001; Mantzoukas \& Jasper, 2004; Offredy et al., 2008; Stubbings et al., 2012). Nursing culture in literature review included rapid response teams and exchange of information in the health care environment (Braaten, 2015; Cappelletti et al., 2014). This study did not explore nursing culture specifically but the teamwork category provided information as to culture of the facility where data collection for this research took place. All participants described seeking the support of charge nurses, rapid response teams, healthcare technicians or physicians. None of the statements in this study included a description of negative responses from these sources of support.

Level of nursing education was described in the literature as mixed related to the effect on nursing practice (Doherty-King \& Bowers, 2013; Martin, 2002; Pardue, 1987; Thompson \& 
Stapley, 2011). This research did not explore the effect of education level on nursing practice. Nurses in this study described experience as more important than education in when providing care to patients experiencing a $\mathrm{CE}$.

Situation awareness and knowledge of the patient were described in the literature as important to decision making (Cappelletti et al., 2014 et al.; Hodge, Hugman, Varndell, \& Howes, 2013; Sedgwick et al., 2014; Tower, Chaboyer, Green, Dyer, \& Wallis, 2012). Consistent with the literature, awareness of patient status had the greatest number of statements in this study with nurses describing various stages of patient awareness in eight subcategories.

Time limits in acute care nursing practice were important in both literature review as well as in this study. Several statements in this study included a focus on time pressure when caring for a patient with a CE. Literature indicates that this is concerning as time pressure decreases decision-making proficiency (Thompson, Dalgleish, et al., 2008).

Thirty-three statements in this study discuss doctors as sources of information, collection of patient information to provide doctors with what they need for decision-making, or the contact when a patient situation deteriorates. None of these statements indicated a negative relationship with the doctors in this facility. The literature review, however, indicate that doctor nurse relationships has positive and negative aspects (Gallagher et al., 2015; Krairiksh \& Anthony, 2001; Merrick, Fry, \& Duffield, 2014). This research did not include interview questions about doctor-nurse interactions.

The Effective Nurse to Nurse Communication framework (ENNC) identified the RSRNs and the RCRNs for this study (Carrington, 2012a). The RSRNs contributed statements associated with awareness of patient status more frequently than the RCRNs possibly indicating that more 
effort toward establishing an understanding of patient condition is needed when nurses do not receive report related to a $\mathrm{CE}$ in patient care. RCRNs provided more statements associated with goals than the RSRNs possibly due to additional information related to the CE from the beginning of the shift. Additional research is required for further identification of these factors and decision-making.

In addition, clinical events (CE) provided the decision-making circumstances on which the nurses would reflect when answering interview questions (Carrington, 2012a). CEs were found in this study to be effective in providing a frame of reference on which the participants could reflect when answering interview questions. Pain as a CE represented half of the CEs experienced by the participants in this study. While pain is not always associated with significant instability in patient condition, it was described by nurses in the hospital in which data collection took place as quite common. When provided with a list of CEs that they would need to reflect on for patient care prior to the beginning of the interview, several nurses made comments similar to “everyone has pain”. Nurses who described caring for patients with lower levels of pain included more options for treatment of pain (ice, repositioning). Nurses who cared for patients who described pain as a " 10 " described medicating patients for pain rather than other options. The high number of pain and varying levels of acuity related to pain level may have influenced the results of this research as not all pain is a "sudden and unexpected change" for this population (Carrington, 2012a).

Several elements of the Naturalistic Decision Making (NDM) framework (Klein, et al., 2010) were found in the categories, themes, and statements in this study. Factors of NDM including time pressure, feedback loops high stakes, multiple players, organizational goals and 
norms, competing goals, uncertainty, and pattern matching (Zsambok, 1997) share similarities with the findings in this research.

For instance, time pressure was described by several nurses in this study. Nurses further described using prioritization and delegation in order to help manage time pressure. Klein et al., (2010) describes intuitive responses to time limited circumstances in decision-making.

Feedback loops are involved when decisions are not made in a single effort (Zsambok, 1997). Nurses responses in this study that resulted in the development of the theme "waiting for patient status to evolve" shared similarities with feedback loops. For instance, one nurse said, "see how things go by the end of the day -- whether she's better or worse or the same, you, kind of, go from there"; P20; RSRN when discussing a patient care circumstance that required decisions, waiting for the outcome of the decision, and then making more decisions.

High stakes decisions are decisions made by decision makers with ties to the outcomes of the decision (Klein et al., 2010). Nurses, similar to the fire ground commanders in Klein et al. (2010) study, are making decisions that can have serious consequences for patients. The nurses in this study did care for patients with serious conditions.

NDM involves multiple players as an important aspect of decision making in high stakes circumstances (Zsambok, 1997). Nurses in this study described collaborating with doctors, nurses, respiratory therapists, and healthcare technicians when discussing caring for patients experiencing CEs. Clearly, the acute care nursing practice environment includes numerous professionals working together to provide patient care.

Organizational goals and norms require decision makers to work within the rules of the environment in which they are making decisions (Zsambok, 1997). Nurses in this study 
described use of nursing protocols, the pain scale for assessment, and other factors in the "following established routine" category. Nurses make decisions with an awareness of rules within their facility.

NDM indicates that high stakes decision makers deal with competing goals leading to a lack of clarity (Zsambok, 1997). Nurses in this study similarly described decision-making challenges such as more than one patient at a time needing nursing care. One nurse stated it this way: “...right in the middle of med pass, it happens -- everything, it always happens at the worst time, --and so, I mean, you just have to -- you can either sit there and reschedule meds or do what you need to do to -- but, I mean, something like that has to be attended to right then and there."; P5 RSRN.

NDM links uncertainty with situations that are real-world and not occurring in the laboratory (Zsambok, 1997). The circumstances of this study involved nurses describing patients they had cared for in an actual hospital environment leading to uncertainty related to patient outcome. One nurse described how she managed uncertainty related to an unknown patient condition: "You're going to want to look at, not only how is she breathing, what is her O2 sat, you know, but what do you see? What do you -- what does she look like? What do you hear? What do her lungs sound like? What does the patient say that she's feeling like and explain what things I've done in the past."; P3 RCRN.

NDM describes decisions made without consideration of options but based on previous similar circumstances (Klein et al., 2010). This led the decision maker to match the current situation to a previous situation and generally only a single option was considered prior to making a decision (Klein et al., 2010). In this study, nurses discussed numerous options related 
to treatment of pain. With more critically ill patients, nurses described calling doctors and charge nurses along with nursing interventions.

Numerous similarities between NDM and the results of this study have been identified. This indicates that NDM is applicable to acute care nursing practice.

\section{Conceptual Framework}

The Communication framework was effective in identifying nursing roles in communication for this research. Bothe RSRNs and RCRNs contributed to all categories. While nurses in this study did not specifically discuss decisions related to developing and communicating nursing report, statements did indicate the importance of report. This represents an important area in science to better support nursing practice.

As intended, this research sought to increase our understanding of decision-making for stages 1 and 4 according to the adapted Effective Nurse-to-Nurse Communication Framework illustrated in Chapter 2. This research project did address these two stages of decision-making by comparing decision-making of responding and receiving nurses.

Factors from Naturalistic Decision Making (NDM) (Klein et al., 2010) did emerge in the categories from this research. This supports the use of NDM in acute care nursing practice to further explore decision-making. Similar to Klein et al (2010), this research did describe important elements used in nurses' decision-making.

\section{Strengths of the Study}

This study was strengthened by a communication framework and NDM theory. Few examples exist in the literature where decision-making was studied by nursing role, caring for patient at the time of event (responding nurse) and continuing care (receiving nurse). This 
approach allowed for varied responses in decision-making by role and revealed shared characteristics by role. This study built on the work of Klein and NDM with a population not previously explored in depth. Nurses, while thought to engage in high stakes decision-making, have not be included in NDM discussions. This study demonstrated that nurses do engage in high stakes decision-making and this study results suggest that additional work is warranted.

\section{Limitations of the Study}

While important elements of nursing decision making emerged in the data, this study did not describe the process that nurses use in their decision-making. The interview was semistructured based on Klein's work and NDM. The results were then constrained to this specific interview. In addition, data collection took place on four units within one hospital. Transferability is limited to similar settings, nurses, and patient circumstances. Triangulation of researchers was limited to two researchers (the author and the dissertation chair). I hypothesize that future work can address these limitations and provide greater insights in the process of nurse decision-making.

The method used to determine differences between RSRN and RCRN nurses facilitated discussion. However, quantitative analysis is needed to examine the significance of these differences within these data.

This research did not use definitions provided by Benner and Tanner (1987) of expert and novice nurses because these definitions were performance based and not based on number of years of acute care experience. This scope of this research did not include an evaluation of nurses' performance to determine if they fit the definitions by Benner and Tanner (1987). Klein et al. (2010) included Fire Ground Commanders (FCG) in their study when exploring factors of 
NDM. The FCG participants had 12 or more years of experience and were included in the study based on their role.

Content analysis provided important information related to the elements of acute care nurse decision-making. However, this method was not in depth enough to explore the processes of decision-making.

\section{Implications for Future Research}

This research describes several aspects of acute care nursing practice that are important to decision-making. Future research using the NDM framework for better understanding of how nurses make decisions in high stakes situations could provide more information related to the value of experienced decision makers in the acute care nursing environment. Nurses in this study did not specifically discuss clinical decision support systems. One nurse did describe an algorithm that popped up when she was caring for a patient following surgery. Five nurses discussed use of protocols in nursing decision-making. As described in chapter four, nurses predominantly chose to ask charge nurses or other healthcare professionals for decision support. More research on the value of the experienced decision maker in nursing practice is warranted.

Awareness of patient status was important to nurses making decisions in this study given the high number of statements falling into this category. More research into how to better support nurses' in their assessment and understanding of patient condition may help support nursing decision making overall.

The complex collaborative environment identified in this study indicates that further understanding of communication between nurses and physicians is needed. Nurses discussed calling physicians to update them on a patient's status, involving charge nurses in decision- 
making, and nursing report. The ENNC addresses communication between nurses during nursing report but future research could explore communication that occurs between nurses and other healthcare professionals.

Future research could explore decision-making and experience more specially associated with role (charge nurse, clinical leader). This could facilitate understanding of nurse decisionmaking based on experience.

Further analysis of the differences between RCRN and RCRN nurses' statements could include quantitative analysis. The number of statements by RSRN (239) and RCRN (190) nurses differed overall as well as within the categories. Statistical analysis of the differences between these groups is required to determine if they are significant.

To improve in methodological approach, future research involving more researchers in the analysis phase would improve rigor. Additional research should be conducted in different facilities and different units to improve transferability for more readers. In data collection, the CE pain should be limited to higher levels for a high stakes decision-making situations. Ideally, this would allow a more even distribution of CEs when exploring decision-making and provide an interview focus as more high stakes when patients with pain are discussed. Processes of decision-making may have emerged, however, content analysis was not able to thoroughly explore the processes. A future secondary analysis using a different method, possibly grounded theory, to examine the data for processes associated with nurse decision-making is warranted.

\section{Summary}

The purpose of this research was to explore nurses' perceptions of clinical decisionmaking for a patient who experienced a clinical event. Acute care nurses were asked questions 
that were based on theory and literature review to aid in the exploration of information important to decision-making. Qualitative content analysis was used to develop Awareness of Patient Status, Nursing Roles outside of specific patient care, Goals, Education/Certification/Hospital Training to support decision-making, Experience and Decision-Making, Time Pressure, Teamwork/Support from Staff, Resources, Following Established Routine. Patient Education, and Consideration of Options to Meet Goals as categories. The results of this research uncover new information for nursing knowledge, provide support for current research, conceptual frameworks, and indicate new directions for future nursing research. 
APPENDIX A:

DATA COLLECTION INTERVIEW GUIDE 
See Table 1 for questions adapted for responding and receiving nurse participants.

- Initial open-ended questions/Grand tour questions

○ "Tell me about an experience you had when caring for a patient in rapid physical decline?" (based on Corbin and Strauss, 2015 unstructured questions)

- Intermediate (more direct seeking deeper explanation) questions: Based on responses from grand tour questions. These questions will be asked as interviews and literature review indicate for richer description of concepts.

○ “Describe events that led up to___ ? ?'(Charmaz, 2014, p. 66)

○ To gather more information on cues: "What were you seeing, hearing, smelling,.." (Klein et al., 1989, p. 466)

○ Knowledge question: "What information did you use in making this decision, and how was it obtained?" (Klein et al., 1989, p. 466)

○ In what ways were you reminded of a previous experience? (L. Phillips, personal communication, March 23, 2015).

○ Goals: "What were you specific goals at the time?" (Klein et al., 1989, p. 466)

○ Options: "What other courses of action were considered by or available to you?" (Klein et al., 1989, p. 466)

○ Basis: "How was this option selected/other options rejected? What rule was being followed?" (Klein et al., 1989, p. 466)

○ Experience: "What specific training, experience or knowledge was necessary or helpful in making this decision?" (Klein et al., 1989, p. 466) 
○ Aiding: "If the decision was not the best, what training, knowledge, or information would have helped?" (Klein et al., 1989, p. 466)

○ Time pressure: "How much time pressure was involved in making this decision?" (Klein et al., 1989, p. 466) (Scale used: 1-5 with 1 being very little time pressure and 5 being immediate decision necessary)

○ Situation Assessment: "Imagine you were asked to describe the situation to a relief nurse at this point, how would you summarize the situation?" (Klein et al., 1989, p. 466)

○ Hypotheticals: "If a key feature of the situation had been different, what difference would it have made in your decision?” (Klein et al., 1989, p. 466).

○ Story telling questions: "Tell me about the last time you...", "Tell me about a particular instance of rapid physical decline that stands out in your mind." (L. Phillips, personal communication, March 13, 2015).

- Potential questions used in second interviews for full concept development:

- "Tell me more about what made XXX stand out as important when caring for this patient?"

- "What were other options besides YYY that you considered when providing care this patient with these symptoms?"

\section{- Ending Questions}

○ "How has this experience helped you grow as a nurse?" (based on Charmaz, 2014) 
○ "After this experience what advice would you give to a less experienced nurse?" (based on Charmaz, 2014)

○ "What is something that you have not thought before that occurred to you during this interview?" (based on Charmaz, 2014)

○ "What else do you think I should know to understand better?" (based on Charmaz, 2014)

○ "What would you like to ask me?" (based on Charmaz, 2014) 
APPENDIX B:

UA IRB APPROVAL LETTER CEDED OVERVIEW 
Date: March 14, 2017

Principal Investigator: Christine Williams Nibbelink

Protocol Number:

1703269939

Protocol Title: Nurse Decision-Making in Acute Care

Level of Review: $\quad$ Administrative Review

Determination: Approved

IRB of Record: Northwest Medical Center

Documents Reviewed Concurrently:

Data Collection Tools: Nibbelink Dissertation Interview Questions.docx HSPP Forms/Correspondence: f204_v2016-07 U of A App for ceded overview.doc HSPP Forms/Correspondence: Nibbelink f107_v2016-07_0 (2).doc

HSPP Forms/Correspondence: Signature page.pdf

Informed Consent/PHI Forms: Aproved Consent form from NW.pdf

Other: Nibbelink CHS Financial Interests in Research Disclosure Form (June 2016 rev).docx

Other: Nibbelink CHS Research Compliance Review Checklist (June 2016).docx

Other: Nibbelink CHS Research Review Request Form (June 2016).docx

Other: Nibbelink Proposal for NW 1.13.17.docx

Other: Nibbelink Template Physician Group Research Services Agreement (June 2015 rev).docx

Other Approvals and Authorizations: Institutional Agreement.pdf

Other Approvals and Authorizations: IRB Approval Letter from NW.pdf

Recruitment Material: $N W$ recruitment flyer.pdf 
Institution Designated the IRB of Record: When an institution is the designated IRB of record, the UA IRB will not review the project. The University of Arizona agrees that it will rely on the review, approval, and continuing oversight by the institution IRB of those protocols approved by the institution pursuant to the terms of the Institutional Review Board Authorization Agreement (if applicable) and as outlined in the HSPP files.

- The University of Arizona maintains a Federalwide Assurance with the Office for Human Research Protections (FWA \#00004218).

- All documents referenced in this submission have been reviewed and are filed with the HSPP.

- The Principal Investigator should notify the IRB immediately:

- Any propososed changes that affect the LOCAL protocol.

- Reports any LOCAL unanticipated problems involving risks to participants or others.

- Continuing Review by the IRB of record has been completed.

- When the study is complete at the LOCAL site.

- Please refer to the Guidance Ceded IRB Review for more information on local PI responsibilities. All research procedures should be conducted according to the approved protocol and the policies and guidance of the IRB of record.

This project has been reviewed and approved by an IRB Chair or designee. 
APPENDIX C:

FACILITY IRB APPROVAL 


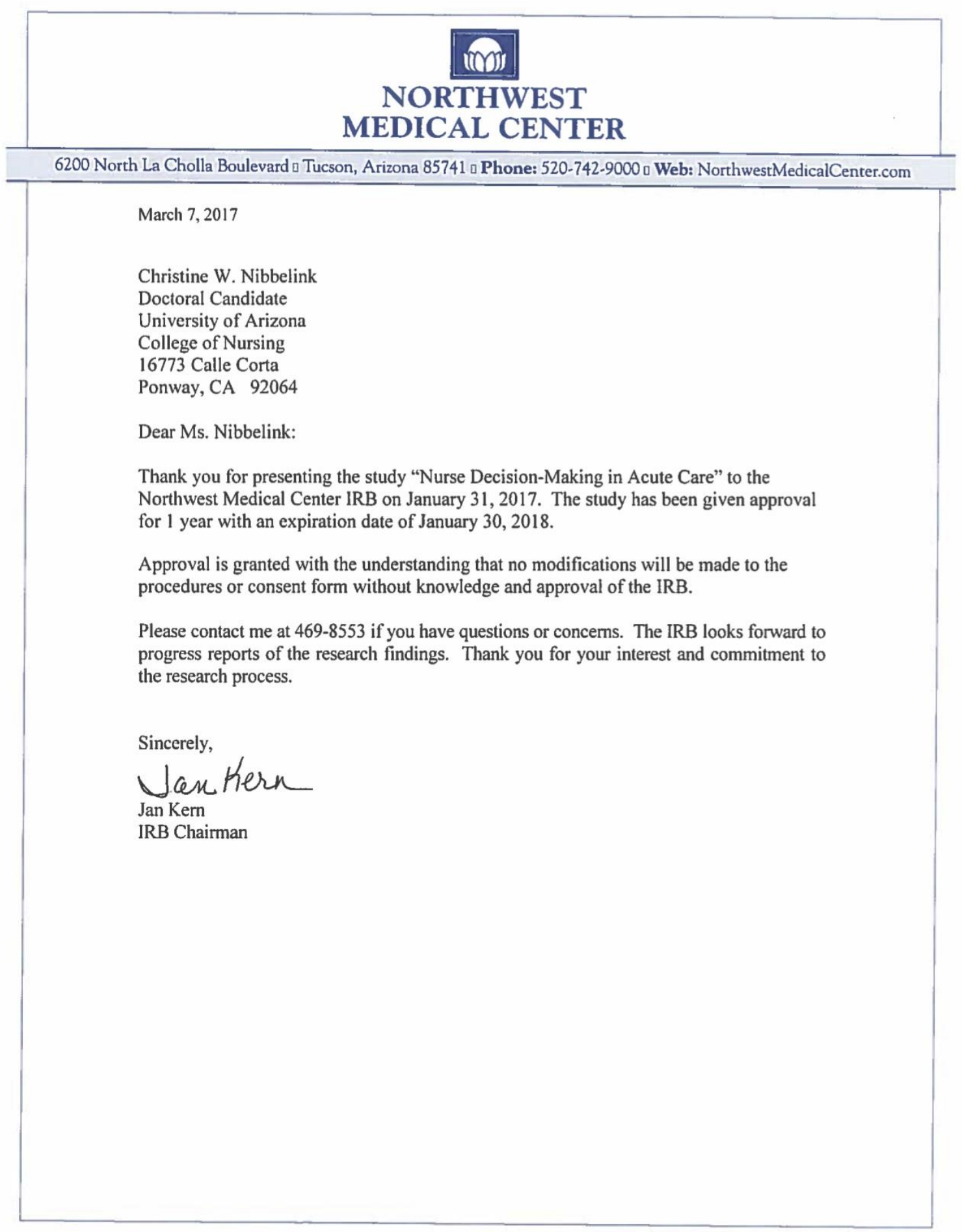


APPENDIX D:

RECRUITMENT FLYER 
Are you a RN who works on Medical or Surgical nursing units?

If so, you may qualify to participate in the study:

"Nurse Decision-Making in Acute Care"

If you are interested or want more information, call or email Christine Nibbelink, RN, MSN

Cell: 970-980-8614 Email: cwnibbelink@email.arizona.edu 
APPENDIX E:

APPROVED CONSENT FOR RESEARCH 


\title{
Consent to Participate in Research
}

Adapted from The University of Arizona Consent to Participate in Research

\author{
Study Title: Nurse Decision-Making in Acute Care \\ Principal Investigator: Christine Nibbelink, MSN, RN \\ Sponsor: Jane Carrington, PhD, RN
}

This is a consent form for research participation. It contains important information about this study and what to expect if you decide to participate. Please consider the information carefully. Feel free to discuss the study with your friends and family and to ask questions before making your decision whether or not to participate.

\section{Why is this study being done?}

The purpose of this research is to explore factors that influence nurse decision making in the acute care setting.

What will happen if I take part in this study?

You will be asked semi-structured open-ended interview questions in a location of your choosing at the hospital. Interviews will be digitally recorded. Participants may choose to not answer a question and that does not result in withdraw from the study. I will begin by asking if you have any questions, turn on the recorder, and begin the interview. Upon conclusion of the interview, the recorder will be turned off, and you will be given a letter of thanks for your personal file. Digitally recorded interviews will then be uploaded to a password protected laptop. The recorder, secured lock box, and laptop will remain in the researcher's possession. All interviews will be de-identified

How long will I be in the study?

Each interview will take 20-30 minutes.

How many people will take part in this study?

Twenty acute care registered nurses are anticipated to participate in this study.

\section{Can I stop being in the study?}

Yes, at any time you may withdraw from the study or refuse to answer questions without risk.

What risks or benefits can I expect from being in the study?

You may feel uncomfortable having their responses recorded. This will be addressed by completing the interviews in a location deemed safe. You may also be assured that data will be de-identified and there is no risk to your employment in participating in this study. By

\begin{tabular}{c} 
DATE: $3-7-17$ EXPIRES: $1-30-18$ \\
\hline APPROVED BY: \\
NORTIIWEST MIEDICAL CENTER IRB \\
THIS STAMP MUST APPEAR ON ALL \\
DOCUMENTS USED TO CONSENT PATIENTS
\end{tabular}


consenting to participate in this study, you are helping nursing science to better understand nurse decision-making in order to better support nursing practice.

Will my study-related information be kept confidential?

Yes, digitally recorded interviews will be uploaded to a password protected laptop. The recorder, secured lock box, and laptop will remain in the researcher's possession. Each interview will be alphanumerically coded for privacy and data organization.

Efforts will be made to keep your study-related information confidential. However, there may be circumstances where this information must be released. If Northwest Medical Center human subjects requests an audit or there is a complaint about the study, then study information will be shared with representatives of the organization.

Also, your records may be reviewed by the following groups:

- The University of Arizona Institutional Review Board

- Office for Human Research Protections or other federal, state, or international regulatory agencies

- The sponsor supporting the study, their agents or study monitors

What are the costs of taking part in this study?

The only anticipated cost to the participant associated with this study is the time required for the interview.

Will I be paid for taking part in this study?

You will receive a $\$ 5$ Starbucks gift card for participating in the study.

Will my data be stored for future research or other uses?

The digital recordings will be stored using an encrypted laptop until all are transcribed and verified verbatim. They will then be deleted. The de-identified text from each interview will be stored on the PI's encrypted laptop and will be used for unspecified future research, publications, and presentations.

Who can answer my questions about the study?

For questions, concerns, or complaints about the study you may contact Christine Nibbelink, cwnibbelink@email.arizona.edu, 970-980-8614.

For questions about your rights as a participant in this study or to discuss other study-related concerns or complaints with someone who is not part of the research team, you may contact the Human Subjects Protection Program at 520-626-6721 or online at http://rgw.arizona.edu/compliance/human-subjects-protection-program. Need NW equivalent?

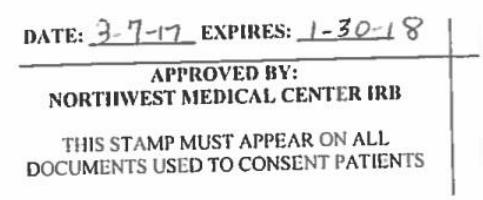


If you are injured as a result of participating in this study or for questions about a study-related injury, you may contact __Christine Nibbelink at cwnibbelink@email.arizona.edu or cell number 970-980-8614

An Institutional Review Board responsible for human subjects research at Northwest Medical Center and found it to be acceptable, according to applicable state and federal regulations and University policies designed to protect the rights and welfare of participants in research.

\section{Signing the consent form}

I have read (or someone has read to me) this form, and I am aware that I am being asked to participate in a research study. I have had the opportunity to ask questions and have had them answered to my satisfaction. I voluntarily agree to participate in this study.

I am not giving up any legal rights by signing this form. I will be given a copy of this form. 
APPENDIX F:

THANK YOU LETTER TO PARTICIPANTS 
To whom it may concern,

Thank you for participating in the study "Nurse Decision-Making in Acute Care" Christine Nibbelink principle investigator. Your interview helps us better understand nurse decision making when faced with a patient experiencing a change in condition or continuing care for the patient.

This letter is for you to use as desired for promotional opportunities or clinical ladder.

Thank you again,

Christine Nibbelink, MSN, RN

Doctoral Candidate

University of Arizona College of Nursing 


\section{REFERENCES}

Agbedia, C. O., Ofi, B., \& Ibeagha, J. E. (2008). Causal model of clinical judgment of practicing nurses, in selected hospitals in Delta State, Nigeria. West African Journal of Nursing, 19(2), 111-120.

Averill, J. B. (2002). Matrix analysis as a complementary analytic strategy in qualitative inquiry. Qualitative Health Research, 12(6), 855-866.

Bakalis, N. (2013). Clinical decision-making in cardiac nursing: a review of the literature. Nursing Standard, 21(12), 39-46.

Benner, P. (2001). From novice to expert. Commemorative Edition. Upper Saddle River, NJ: Prentice Hall.

Benner, P., Hughes, R. G., \& Sutphen, M. (2008). Clinical reasoning, decision making and action: thinking critically and clinically. In R. G. Hughes (Ed.), Patient safety and quality: an evidence-based handbookfor nurses. Rockville, MD: Agency for Healthcare Research and Quality.

Benner, P. \& Tanner, C. (1987). Clinical judgment: how expert nurses use intuition. Am J Nurs, 87(1), 23-31.

Blumer H. (1969). Symbolic interactionism: perspective and method. Berkeley, CA: University of California Press.

Braaten, J. S. (2015). Hospital system barriers to rapid response team activation: a cognitive work analysis. American Journal of Nursing, 115(2), 22-33.

doi:10.1097/01.NAJ.0000460673.82070.af

Bruno, C. (2013). Understanding symptom experiences of older individuals with acute coronary syndrome. University of Arizona: Tucson, AZ.

Bucknall, T. K. (2000). Critical care nurses' decision-making activities in the natural clinical setting. J Clin Nurs, 9(1), 25-35.

Cappelletti, A., Engel, J. K., \& Prentice, D. (2014). Systematic review of clinical judgment and reasoning in nursing. Journal of Nursing Education, 53(8), 453-458.

doi:10.3928/01484834-20140724-01

Carrington, J. M. (2008). The effectiveness of the electronic health record with standardized nursing languages for communicating patient status related to a clinical event. Dissertation Abstracts International, 69(03), AAT3297974. 
Carrington, J. M. (2012a). Development of a conceptual framework to guide a program of research exploring nurse-to-nurse communication. Comput Inform Nurs, 30(6), 293-299. doi:10.1097/NXN.0b013e31824af809

Carrington, J. M. (2012b). The usefulness of nursing languages to communicate a clinical event. Comput Inform Nurs, 30(2), 82-88; quiz 89-90. doi:10.1097/NCN.0b013e318224b338

Carrington, J. M. \& Effken, J. A. (2011). Strengths and limitations of the electronic health record for documenting clinical events. Comput Inform Nurs, 29(6), 360-367. doi:10.1097/NCN.0b013e3181fc4139

Carvalho, P. V. R., dos Santos, I. L., \& Vidal, M. C. R. (2005). Nuclear power plant shift supervisor's decision making during microincidents. International Journal of Industrial Ergonomics, 35(7), 619-644. doi:10.1016/j.ergon.2005.01.010

Charmaz, K. (2014). Constructing grounded theory (2nd ed.). Los Angeles, CA: Sage.

Cioffi, J. (2012). Expanding the scope of decision-making research for nursing and midwifery practice. Int J Nurs Stud, 49(4), 481-489. doi:10.1016/j.ijnurstu.2011.10.015

Coiera, E. (2003). Clinical decision support systems. In Guide to Health Informatics $\left(2^{\text {nd }} e d.\right)$ (p. 417-432). Oxford University Press: New York, NY.

Corbin, J. \& Strauss, A. (2015). Basics of qualitative research: Techniques and procedures for developing grounded theory (4th ed.). Los Angeles, CA: Sage.

Corcoran, S. A. (1986). Task complexity and nursing expertise as factors in decision making processes used by nurses to plan patient care. Nurs Res, 35(2), 107-112.

Crotty, M. (1998). The foundations of social research. Los Angeles: Sage.

Denzin, N. K. \& Lincoln, Y. S. (2000). Handbook of qualitative research. Thousand Oaks, CA: Sage Publications.

Doherty-King, B. \& Bowers, B. J. (2013). Attributing the responsibility for ambulating patients: a qualitative study. Int J Nurs Stud, 50(9), 1240-1246.

Dougherty, L., Sque, M., \& Crouch, R. (2012). Decision-making processes used by nurses during intravenous drug preparation and administration. J Adv Nurs, 68(6), 1302-1311. doi:10.1111/j.1365-2648.2011.05838.x

Downing, J., Yoder, L. H., \& Kirksey, K. M. (2011). A qualitative study of phlebotomy device selection. MEDSURG Nursing, 20(6), 291-295.

Endsley, M. R. (1995). Measurement of situation awareness in dynamic systems. Human Factors: The Journal of Human Factors and Ergonomic Society, 37(1), 65-84. 
Endsley, M. R. (1997). The role of situation awareness in naturalistic decision making. In C. E. Zsambok, Klein, G. (Ed.), Naturalistic decision making (pp. 269-283). Mahwah, NJ: Lawrence Erlbaum Associates.

Fonseca, L. F. \& Brennan, P. F. (2012). Computerized decision support system and decision quality of nurses. Ciencia, Cuidado e Saude, 11, 267-273.

Forbes, A. G., Surdeneau, M., P. Jansen, \& Carrington, J. M. (In press). Transmitting narrative: designing an interactive shift-summarization tool for improving nurse communication. In IEEE Workshop on Interactive Visual Text Analytics, Atlanta, Georgia, October, 2013.

French, B. (2006). Uncertainty and information need in nursing. Nurse Education Today, 26(3), 245-252

Fry, M., \& MacGregor, C. (2014). Confidence and impact on clinical decision-making and behaviour in the emergency department. Australasian Emergency Nursing Journal, 17(3), 91-97. doi:10.1016/j.aenj.2014.03.003

Gallagher, A., Bousso, R. S., McCarthy, J., Kohlen, H., Andrews, T., Paganini, M. C., . . . Padilha, K. G. (2015). Negotiated reorienting: a grounded theory of nurses' end-of-life decision-making in the intensive care unit. Int J Nurs Stud, 52(4), 794-803. doi:10.1016/j.ijnurstu.2014.12.003

Glesne, C. (2011). Becoming qualitative researchers: an introduction (4th ed.) New York: Pearson.

Goodwin, L. D. \& Goodwin, W. L. (1985). Statistical techniques in "AERJ" articles, 1979-1983: the preparation of graduate students to read the educational research literature. Educational Researcher, 14(2), 5-11.

Gray, L. M. \& Meyer, S. (2014). Management of patients on chemotherapeutic treatment for advanced cancer with acute conditions in the emergency department. Australasian Emergency Nursing Journal, 17(4), 146-151. doi:http://dx.doi.org/10.1016/j.aenj.2014.05.003

Guba, E. G. \& Lincoln, Y. S. (1982). Epistemological and methodological bases of naturalistic inquiry. Educational Communication and Technology, 30(4), 233-252.

Hagbaghery, M. A., Salsali, M., \& Ahmadi, F. (2004). The factors facilitating and inhibiting effective clinical decision-making in nursing: a qualitative study. BMC Nurs, 3(1), 2. doi:10.1186/1472-6955-3-2

Hedberg, B. \& Larsson, U. S. (2003). Observations, confirmations and strategies -- useful tools in decision-making process for nurses in practice? J Clin Nurs, 12(2), 215-222. doi:10.1046/j.1365-2702.2003.00703.x 
Hendry, C. \& Walker, A. (2004). Priority setting in clinical nursing practice: literature review. $J$ Adv Nurs, 47(4), 427-436. doi:10.1111/j.1365-2648.2004.03120.x

Hodge, A., Hugman, A., Varndell, W., \& Howes, K. (2013). A review of the quality assurance processes for the Australasian Triage Scale (ATS) and implications for future practice. Australasian Emergency Nursing Journal, 16(1), 21-29. doi:10.1016/j.aenj.2012.12.003

Hoffman, K., Donoghue, J., \& Duffield, C. (2004). Decision-making in clinical nursing: investigating contributing factors. $J A d v$ Nurs, 45(1), 53-62.

Holl, R. M. (1996). Independent patient care decisions in the hospital and staff nurse characteristics. Journal of Nursing Science, 1(5/6), 148-156

Husted, G. L. (2001). The feelings nurses and patients/families experience when faced with the do-not-resuscitate status: conflict and culture brokering in critical care units. Heart Lung, 23(6), 458-465.

Kihlgren, A., Forslund, K., \& Fagerberg, I. (2006). Managements' perception of community nurses' decision-making processes when referring older adults to an emergency department. J Nurs Manag, 14(6), 428-436. doi:10.1111/j.1365-2934.2006.00642.x

Klein, G. \& Klinger, D. (1991). Naturalistic decision making. Gateway, 11(3), 16-19.

Klein, G. A. (1993). A recognition-primed decision (RPD) model of rapid decision making. In G. A. Klein, Orasanu, J., Calderwood, R., \& Zsambok, C. E. (Ed.), Decision making in action: models and methods (pp. 138-147). Norwood, New Jersey: Ablex Publishing Company.

Klein, G. A. \& Calderwood, R. (1991). Decision models: some lessons from the field. IEEE transactions on systems, man, and cybernetics, 21(5), 1018-1026. doi:10.1109/21.120054

Klein, G., Calderwood, R., \& Clinton-Cirocco, A. (2010). Rapid decision making on the fire ground: the original study plus a postscript. Journal of Cognitive Engineering and Decision Making, 4(3), 186-209. doi:10.1518/155534310X12844000801203

Klein, G. A., Calderwood, R., \& MacGregor, D. (1989). Critical decision method for eliciting knowledge. IEEE transactions on systems, man, and cybernetics, 19(3), 462-472. doi:10.1109/21.31053

Klein, G., Wolf, S., Militello, L., \& Zsambok, C. (1995). Characteristics of skilled option generation in chess. Organizational behavior and human decision processes, 62(1), 6369. doi:10.1006/obhd.1995.1031

Krairiksh, M. \& Anthony, M. K. (2001). Benefits and outcomes of staff nurses' participation in decision making. Journal of Nursing Administration, 31(1), 16-23. 
Krippendorff, K. (2003). Content analysis: an introduction to its methodology (3rd ed.). Los Angeles, CA: Sage.

Kohn, L. T., Corrigan, J. M., \& Donaldson, M. S. (1999). Errors in health care: a leading cause of death and injury. To err is human: building a safer health system (pp. 26-48). Retrieved from http://www.nap.edu/download.php?record_id=9728\#

Lincoln, Y. \& Guba, E. (1985). Naturalistic inquiry. Beverly Hills, CA: Sage.

Lipshitz, R., Klein, G., Orasanu, J., \& Salas, E. (2001). Taking stock of naturalistic decision making. Journal of Behavioral Decision Making, 14(5), 331-352. doi:10.1002/bdm.381

Mantzoukas, S. \& Jasper, M. A. (2004). Reflective practice and daily ward reality: a covert power game. J Clin Nurs, 13(8), 925-933. doi:10.1111/j.1365-2702.2004.01008.xneed to make bioethical decisions. Nursing Administration Quarterly, 25(3), 46-54.

Marshall, A. P., West, S. H., \& Aitken, L. M. (2013). Clinical credibility and trustworthiness are key characteristics used to identify colleagues from whom to seek information. J Clin Nurs, 22(9/10), 1424-1433. doi:10.1111/jocn.12070

Martin, C. (2002). The theory of critical thinking of nursing. Nurs Educ Perspect, 23(5), 243247.

Mead, H. (1967). Mind, self, society from the standpoint of a social behaviorist from the standpoint of a social behaviorist. C. W. Morris (ed.). In Mind, self, society from the standpoint of a social behaviorist from the standpoint of a social behaviorist. Chicago, IL: University of Chicago Press.

Miles, M., Huberman, A. M., \& Saldana, J. (2014). Qualitative data analysis: a sourcebook. (3rd ed.). Thousand Oaks, CA: Sage Publications.

Miles, M., Huberman, A. M., \& Saldana, J. (1994). An expanded sourcebook: qualitative data analysis. (2nd ed.). Thousand Oaks, CA: Sage Publications.

Merrick, E., Fry, M., \& Duffield, C. (2014). Australian practice nursing: collaboration in context. J Clin Nurs, 23(23/24), 3525-3532. doi:10.1111/jocn.12605

Mrayyan, M. T. (2004). Nurses' autonomy: influence of nurse managers' actions. $J$ Adv Nurs, 45(3), 326-336. doi:10.1046/j.1365-2648.2003.02893.x

Munhall, P. L. (2012). Nursing research: a qualitative perspective (5th ed.). Sudbury, MA.: Jones \& Bartlett Learning.

Offredy, M., Kendall, S., \& Goodman, C. (2008). The use of cognitive continuum theory and patient scenarios to explore nurse prescribers' pharmacological knowledge and decisionmaking. Int J Nurs Stud, 45(6), 855-868. doi:10.1016/j.ijnurstu.2007.01.014 
Panniers, T. L. \& Walker, E. K. (1994). A decision-analytic approach to clinical nursing. Nurs Res, 43(4), 245-249.

Pantazopoulos, I., Tsoni, A., Kouskouni, E., Papadimitriou, L., Johnson, E. O., \& Xanthos, T. (2012). Factors influencing nurses' decisions to activate medical emergency teams. J Clin Nurs, 21(17/18), 2668-2678. doi:10.1111/j.1365-2702.2012.04080.x

Pardue, S. F. (1987). Decision-making skills and critical thinking ability among associate degree, diploma, baccalaureate, and master's-prepared nurses. J Nurs Educ, 26(9), 354-361.

Parker, C. G. (2014). Decision-making models used by medical-surgical nurses to activate rapid response teams. Medsurg Nurs, 23(3), 159-164

Patton, M. Q. (2002). Qualitative research and evaluation methods (3rd ed.). Thousand Oaks: Sage.

Phipps, D. L. \& Parker, D. (2014). A naturalistic decision-making perspective on anaesthetists' rule-related behaviour. Cognition, Technology \& Work, 16(4), 519-529. doi:10.1007/s10111-014-0282-2

Radwin, L. E. (1998). Empirically generated attributes of experience in nursing. $J$ Adv Nurs, 27(3), 590-595. doi:10.1046/j.1365-2648.1998.00548.x

Randel, J. M., Pugh, H. L., \& Reed, S. K. (1996). Differences in expert and novice situations awareness in naturalistic decision making. International Journal of Human-Computers Studies, 45(5), 579-597.

Rycroft-Malone, J., Fontenla, M., Seers, K., \& Bick, D. (2009). Protocol-based care: the standardisation of decision-making? J Clin Nurs, 18(10), 1490-1500.

Samuriwo, R. \& Dowding, D. (2014). Nurses' pressure ulcer related judgements and decisions in clinical practice: A systematic review. Int J Nurs Stud, 51(12), 1667-1685. doi:http://dx.doi.org/10.1016/j.ijnurstu.2014.04.009

Sandelowski, M. (2000). Focus on research methods. Whatever happened to qualitative description? Research in Nursing \& Health, 23(4), 334-340 337p.

Sandelowski, M. (2010). What's in a name? Qualitative description revisited. Research in Nursing \& Health, 33(1), 77-84 78p. doi:10.1002/nur.20362

Sandelowski, M. (1995). Focus on qualitative methods. Sample size in qualitative research. Research in Nursing \& Health, 18(2), 179-183.

Sandelowski, M. (1986). The problem of rigor in qualitative research. ANS Adv Nurs Sci, 8(3), 27-37. 
Sandelowski, M. (1993). Rigor or rigor mortis: the problem of rigor in qualitative research revisited. ANS Adv Nurs Sci, 16(2), 1-8.

Sandroni, C., Ferro, G., Santangelo, S., Tortora, F., Mistura, L., Cavallaro, F., .. A Antonelli, M. (2004). In-hospital cardiac arrest: survival depends mainly on the effectiveness of the emergency response. Resuscitation, 62(3), 291-297. doi:

10.1016/j.resuscitation.2004.03.020

Sedgwick, M. G., Grigg, L., \& Dersch, S. (2014). Deepening the quality of clinical reasoning and decision-making in rural hospital nursing practice. Rural Remote Health, 14(3), 2858.

Seright, T. J. (2011). Clinical decision-making of rural novice nurses. Rural Remote Health, 11(3), 1726.

Shannon, C. E. (1967). The mathematical theory of communication. In C. E. Shannon \& W. Weaver (Eds.). The mathematical theory of communication. Chicago IL: University of Illinois Press.

Spooner, S. A. (2007) Mathmatical foundations of decision support systems. In E. S. Berner (Ed.), Clinical Decision Support Systems, (2nd ed), (p. 19-43). New York: SpringerVerlag.

Spradley, J. P. (1980). Participant observation. Fort Worth, TX: Harcourt College Publishers.

Stewart, J., Stansfield, K., \& Tapp, D. (2004). Clinical nurses' understanding of autonomy: accomplishing patient goals through interdependent practice. Journal of Nursing Administration, 34(10), 443-450.

Stubbings, L., Chaboyer, W., \& McMurray, A. (2012). Nurses' use of situation awareness in decision-making: an integrative review. J Adv Nurs, 68(7), 1443-1453. doi:10.1111/j.1365-2648.2012.05989.x

Tanner, C. A. (2006). Thinking like a nurse: a research-based model of clinical judgment in nursing. J Nurs Educ, 45(6), 204-211.

Thompson, C., Bucknall, T., Estabrookes, C. A., Hutchinson, A., Fraser, K., de Vos, R., . . . Saunders, J. (2009). Nurses' critical event risk assessments: a judgement analysis. J Clin Nurs, 18(4), 601-612. doi:10.1111/j.1365-2702.2007.02191.x

Thompson, C., Spilsbury, K., Dowding, D., Pattenden, J., \& Brownlow, R. (2008). Do heart failure specialist nurses think differently when faced with 'hard' or 'easy' decisions: a judgement analysis. J Clin Nurs, 17(16), 2174-2184. doi:10.1111/j.13652702.2008.02303.x 
Thompson, C., \& Stapley, S. (2011). Do educational interventions improve nurses' clinical decision making and judgement? A systematic review. Int J Nurs Stud, 48(7), 881-893. doi:http://dx.doi.org/10.1016/j.ijnurstu.2010.12.005

Tower, M., Chaboyer, W., Green, Q., Dyer, K., \& Wallis, M. (2012). Registered nurses' decisionmaking regarding documentation in patients' progress notes. J Clin Nurs, 21(19-20), 2917-2929. doi:10.1111/j.1365-2702.2012.04135.x

Venes, D. (Ed.). (2005). Taber's cyclopedic medical dictionary (21st ed.). Philadelphia: F. A. Davis Company.

Walker, L. O. Avant, K. C. (2011). Strategies for theory construction in nursing (5th ed.). Boston, MA.: Prentice Hall.

Sandroni, C., Ferro, G., Santangelo, S., Tortora, F., Mistura, L., Cavallaro, F., .. Antonelli, M. (2004). In-hospital cardiac arrest: survival depends mainly on the effectiveness of the emergency response. Resuscitation, 62(3), 291-297. doi:10.1016/j.resuscitation.2004.03.020

Zsambok, C. E. (1997). Naturalistic decision making: Where are we now? In C. E. Zsambok \& G. Klein (Eds.), Naturalistic decision making (p. 3-16). Mahwah, N.J.: Lawrence Erlbaum Associates. 